\title{
Avoiding the Uncanny Valley - Robot Appearance, Personality and Consistency of Behavior in an Attention-Seeking Home Scenario for a Robot Companion
}

\author{
Michael L. Walters, Dag S. Syrdal, Kerstin Dautenhahn, René te Boekhorst, Kheng Lee Koay. ${ }^{i}$ \\ Adaptive Systems Research Group, School of Computer Science, University of Hertfordshire, College Lane, \\ Hatfield, Herts. UK. \\ Email: \{M.L.Walters, D.S.Syrdal, K.Dautenhahn, R.teBoekhorst, K.L.Koay\}@herts.ac.uk
}

\begin{abstract}
This article ${ }^{\mathrm{ii}}$ presents the results of video-based Human Robot Interaction (HRI) trials which investigated people's perceptions of different robot appearances and associated attention-seeking features and behaviors displayed by robots with different appearance and behaviors. The HRI trials studied the participants' preferences for various features of robot appearance and behavior, as well as their personality attributions towards the robots compared to their own personalities. Overall, participants tended to prefer robots with more human-like appearance and attributes. However, systematic individual differences in the dynamic appearance ratings are not consistent with a universal effect. Introverts and participants with lower emotional stability tended to prefer the mechanical looking appearance to a greater degree than other participants. It is also shown that it is possible to rate individual elements of a particular robot's behavior and then assess the contribution, or otherwise, of that element to the overall perception of the robot by people. Relating participants' dynamic appearance ratings of individual robots to independent static appearance ratings provided evidence that could be taken to support a portion of the left hand side of Mori's theoretically proposed 'uncanny valley' diagram. Suggestions for future work are outlined.
\end{abstract}

\section{I.INTRODUCTION}

Robots that are currently commercially available for use in a domestic environment and which have human interaction features are often orientated towards toy or entertainment functions. In the future, a robot companion which is to find a more generally useful place within a human oriented domestic environment, and thus sharing a private home with a person or family, must satisfy two main criteria (Dautenhahn et al. (2005); Syrdal et al. (2006); Woods et al. (2007)):

It must be able to perform a range of useful tasks or functions.

It must carry out these tasks or functions in a manner that is socially acceptable and comfortable for people it shares the environment with and/or it interacts with.

The technical challenges in getting a robot to perform useful tasks are extremely difficult, and many researchers are currently researching into the technical capabilities that will be required to perform useful functions in a human centered environment including navigation, manipulation, vision, speech, sensing, safety, system integration and planning. The second criteria is arguably equally important, because if the robot does not exhibit socially acceptable behavior, then people may reject the robot if it is annoying, irritating, unsettling or frightening to human users. Therefore:

\section{How can a robot behave in a socially acceptable manner?}

Research into social robots is generally contained within the rapidly developing field of Human-Robot Interaction (HRI). For an overview of socially interactive robots (robots designed to interact with humans in a social way) see Fong et al. (2003). Relevant examples of studies and investigations into human reactions to robots include: Goetz et al. (2003) where issues of robot appearance, behavior and task domains were investigated, and Severinson-Eklundh et al. (2003) which documents a longitudinal HRI trial investigating the human perspective of using a robotic assistant over several weeks. Khan (1998), Scopelliti et al. (2004) and Dautenhahn et al. (2005) have surveyed peoples' views of domestic robots in order to aid the development of an initial design specification for domestic or servant robots. Kanda et al. (2004) presents results from a longitudinal HRI trial with a robot as a social partner and peer tutor aiding children learning English. 


\section{II.RELATED WORK}

\section{INTRODUCTION TO RELATED WORK}

As is often the case in HRI, due to its multidisciplinary nature (Sabanovic et al. (2007)), the theoretical and empirical bases for this study draws on three different, yet interlocking fields, which will be discussed separately in three sections below. The first section will draw upon observations from HRI research on the impact of robot appearance. This section is followed by a short summary from psychological and HRI literature on how humans make attributions as to personality, intentions and capabilities of both humans and robots in situations based on appearance, especially in situations with limited information available. Finally, the possible impact of individual differences on related to robot appearance, as well as in HRI in general, will be examined, before concluding this section with the presentation of the research questions concerning the current study.

\section{A.Appearance and Behavior Matter}

As is the case for human-human interaction (cf. Zebrowitz et al. (2004)), it is to be expected that human perceptions of robot social behavior will depend to a large extent on its appearance. Robots may be placed on an anthropomorphic appearance scale which varies from mechanical-looking to a human-like appearance as suggested by Kiesler \& Goetz (2002), Goetz et al. (2003) and Woods et al. (2004). Hinds et al. (2004) have studied the effect of robot appearance on humans carrying out a joint task with a robot. Findings show that mechanical-looking robots tend to be treated less politely than robots with a more human-like appearance. Also, humans commonly treat mechanical-looking robots in a subservient way (i.e. less socially interactively) compared to more human-looking robots. Moreover, expectations are in general lower with regard to abilities and reliability for mechanical-looking robots. Most currently commercially available research robots tend to have a somewhat mechanical appearance, though some have incorporated various simple humanoid features such as arms, faces, eyes and so on. Some research robots, often referred to as androids, are very human-like in appearance, though, at present, their movements and behavior falls far short of emulating that of real humans. Mori (1970) proposed a general effect in which people will act in a more familiar way towards robots as they exhibit increasingly human-like characteristics. However at a certain point the effect becomes repulsive due to robots that look very human-like, but their behavior identifies them as robots. This proposed effect can be illustrated by means of Mori's diagram (see Figure 1) where the shape of the curves gives rise to the term 'uncanny valley' to describe the repulsive effect. Mori's original proposal indicated that the 'uncanny valley' effect was present for inanimate likenesses, but was even more pronounced for robots, puppets and automata which actually exhibit movement. Therefore, according to Mori, although robot appearance is important with regard to familiarity and social acceptance, the actual quality and content of a robot's movements may be even more important. Mori argued that robot appearance and behavior must be consistent with each other. At the extreme of high fidelity appearance, even slight inconsistencies in behavior can have a powerful unsettling effect. Note, the 'uncanny valley' effect is not uncontroversial, though some roboticists such as Ferber (2003) and Hanson et al. (2005), have argued that there is conflicting evidence for the right hand side of Mori's "Uncanny Valley" diagram. Hanson (2006) has more recently proposed that even non-human-like robots with an abstract appearance can exhibit the uncanny effect if the "aesthetic is off" in a similar way that may occur with cosmetically atypical humans. Bethal \& Murphy (2006) found that when a mechanical looking rescue robot has approached trapped people, many of the trapped people have have reported that they perceived the robot as "creepy" and unnerving. To tackle this problem Bethal and Murphy have identified some ways in which mechanical looking (search and rescue) robots can use appropriate 'body movements' and postures to provide useful non-verbal expressions of non-threatening and re-assuring behavior to (trapped) humans being rescued. That these mechanical looking rescue robots can sometimes appear threatening to (non-familiar) humans also lends some credence to Hanson's assertions that non-human-like robots can also exhibit 'uncanny' characteristics (Hanson (2006)).Much anecdotal evidence from practitioners in CGI (Computer-generated imagery), film effects and sculpture also seems to support Mori's original conjecture on the uncanny valley and the possible underlying reasons are discussed in Brenton et al. (2005). Research is continuing into the area of very human-like robots or androids. For example, Minato et al. (2004) and Ishiguro (2007) have built android robots a) to study how humans interact with robots which have a very human-like appearance and b) in order to gain insights into human cognition.

Inspired by Mori's original theory, Minato et al. (2004a) have proposed that if a particular robot's appearance and associated behavior were consistent and more human like, but not to the extent that the 'uncanny valley' was reached, it would be more acceptable and effective at interacting with people (cf. MacDorman (2005) \& Woods et al. (2004)). However, Goetz et al. (2003) also argued that appearance should be matched to the type of task that a robot is to perform, and as such the degree of human-likeness which is desirable for any given role or task may vary. Socially acceptable robot behavior therefore encompasses various issues related to robot appearance and behavior. Lee \& Keisler (2005) have examined how humans form a 'mental model' of a (humanoid) robot by making unconscious assumptions from the robots attributes and features within a very short period of exposure. The mental model is then used by the human as a guide as to 
the likely state, knowledge, capabilities and performance to be expected from a particular robot. If the appearance and the behavior of the robot are more advanced than the true state of the robot, then people will tend to judge the robot as dishonest as the (social) signals being emitted by the robot, and unconsciously assessed by humans, will be misleading. On the other hand, if the appearance and behavior of the robot are unconsciously signaling that the robot is less attentive, socially or physically capable than it actually is, then humans may misunderstand or not take advantage of the robot to its full abilities. Kanda et al. (2007) has investigated some of these issues with a robot which (pretended to) listen to a human user when the human is providing route directions to the robot. When the robot exhibited social behaviors, such as eye contact, head nodding and arm movements, the users had a greater impression of the robot paying attention, of reliability and sympathy, and understanding the directions correctly than when the robot did not exhibit these behaviors. In this case, however, the robot was not listening to the human giving directions at all and therefore was in fact misleading! In a comparable real life situation, a robot could advantageously make appropriate social (behavior) signals to reinforce or indicate that it had genuinely understood correctly or otherwise.

A theme that recurs in all these arguments and speculations about Mori's 'uncanny valley', task domains, robot appearance and social behavior is the desirability of consistency between the robot appearance and behavior. That is, the robot should behave both socially, and also with regard to functional and task capabilities, in a way which is consistent with its appearance. In a previous study where robots interacted with children in a game scenario, we have found that the children's attention is attracted more by a robot's pointing arm and camera movement when they are consistent with each other (te Boekhorst et al. (2006). Bruce et al. (2002) studied a robot that exhibited both appearance (a facial expression) and behavior (turning movement) cues. Findings showed that the cues were more effective at gaining peoples attention when both were used together consistently together.

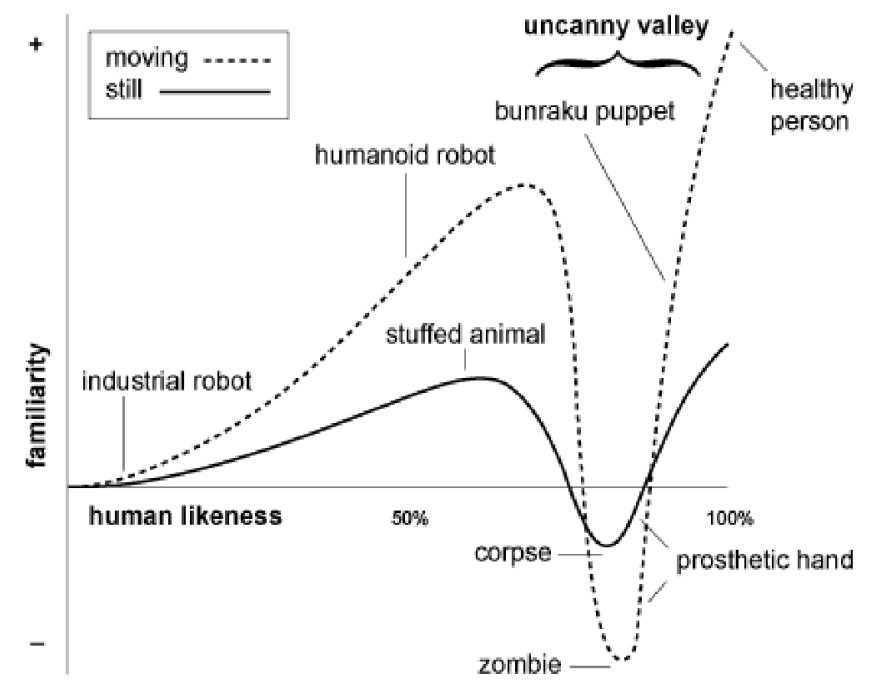

Figure 1. Mori's uncanny valley diagram (simplified and translated by MacDorman (2005)-GFDL).

Research has shown that humans do respond to social characteristics, features or behaviors exhibited by less human-like robots. Reeves \& Nass (1996) showed that in interaction with computer technology people exhibit aspects of social behavior towards computers. Computer based technology and systems contribute to a large part of most current robots, so it is expected that people will perceive and respond to robots in similar social ways as they do to computers. Many researchers have demonstrated such 'social behaviour' that people may exhibit towards robots, e.g. work by Breazeal (2002) has shown that people tend to engage more actively with robots that possess relatively simple expressive capabilities. However, people do not treat robots exactly as they treat each other: A study by Friedman et al. (2003) has shown that while people in many ways view an Aibo (dog like) robot like a real dog, they do not treat it, and do not view it in precisely the same way as a living dog (e.g. with regard to moral standing). In our previous work (cf. Walters et al. (2005a)) we have found that although a majority of humans approach mechanical looking robots to spatial distances which seem to correspond to Hall's social distances (cf. Hall (1966) \& Hall (1968)), a sizable minority were observed to take up a close positions which would be seen as either intimate or threatening if observed in human-human interactions according to Hall's spatial zone theory. However, as long as robots can still be distinguished from biological organisms, which may be the case for a long time to come, it is unlikely that people will react socially to robots in exactly the same ways that they might react to other humans or other living creatures in comparable contexts (cf. Khan (1998), Norman (2001), Dryer (1999), Dautenhahn (2002), \& Dautenhahn et al. (2006)). 


\section{B.Human Perception of Robot Appearance and Robot Personality}

An important influence on how humans perceive and interact socially with other humans is by assessing the personality of other humans they meet (often at very short acquaintance) and extrapolating from the various (stereotypical) personality traits that they may observe in others. Lee \& Kiesler (2005) have observed a similar process taking place with regard to the formation of humans' mental models of robots, and it is interesting to us to see if the same mechanisms of (robot) personality attribution contribute to the formation of mental models of robots in a similar way. The anthropomorphic attribution of personality to non-human entities can be viewed in two separate ways. One way is to view it as a fallacy that obscures the reality of behavior that does not correspond to human behavior and thus impedes progress towards understanding and prediction of non-human behavior (Davis, 1997). The other is to see such attribution as a useful heuristic in describing behavior in terms that are relatively easily understood by most audiences (Asquith, 1997). Nass \& Reeves (Nass et al., 1995a; Nass et al., 1995b; Reeves \& Nass (1996)) suggest that this attribution of personality to a wide range of technological artifacts (including computers and robots) is difficult to avoid. They also state that utilizing this phenomenon can be helpful when designing user interfaces and behavior of such artifacts as it allows the easy and intuitive prediction of system behavior by the user. This notion has been commonly adopted in the design and use of virtual agents, where consistent agent personality, often analogous to human personality have been used a means to create a more intuitive and engaging interface (Ball \& Breeze, 2000). This argument is also presented by Duffy (2003) and applied to HRI. In the domain of HRI, when confronted with entities with unknown behavior, such as robots, anthropomorphism might thus be used as a guide to cope with the unpredictability of the situation. The implications of such a paradigm is that robot design should endeavor to create robot appearances to which personality attributions are made that correspond to the intended behavior of the robot as suggested by Goetz et al. (2003). For this to be possible, it is necessary to explore the relationship between personality attribution and appearance, in HRI situations. To be able to fully explore this relationship we will first consider how humans rate other humans in terms of personality with limited information before we investigate HRI studies.

In the field of personality and social psychology, studies have investigated how successfully participants rate strangers on various personality dimensions at zero acquaintance, for example, in contexts in which perceivers are given no opportunity to interact with 'strangers' (targets of whom no prior knowledge is available to the subject) (Albright et al., 1988). These studies found that the traits of Extroversion, Agreeableness and Conscientiousness seem to allow for the most successful rating of strangers, with Emotional Stability and Openness to Experience the most difficult to rate (Albright et al., 1988; Borkenau \& Liebler, 1992). This effect is exhibited even in situations where there is no interaction between participants and even when rating is done purely on the basis of emails (Gill et al., 2006). This body of research also revealed that Extroversion ratings are highly correlated with the physical attractiveness ratings of the person being rated. Of particular interest for our experiment is the Borkenau \& Liebler (1992) study where participants rated strangers according to the Big Five personality traits (see section II.B.) after having only either seen still photos or videos of the strangers. If one purely extrapolates the results from human-human studies on personality attributions to HRI one would expect that Extroversion, Conscientiousness and Agreeableness will be the personality traits with the largest systematic variance in participant ratings due to cues arising from appearance and behavior. It would therefore be expected that these are the traits where people's ratings will change the most according to differences in between robots. Research on the attribution of personality to robots does to some extent support this extrapolation. Kiesler \& Goetz (2002) reported that participants found it easier to rate the robot on the extroversion dimension, while finding Emotional Stability and Openness to experience the most difficult dimensions in which to rate the robot. Yan et al. (2004) also found that when varying AIBO behavior along the extroversion - introversion dimension, participants were able to distinguish between the two personality types.

Note, our previous study (Woods et al. 2006) also investigated the issue of participants projecting their personality traits onto the robot but found that this was not the case. This will, however, not be the focus of this paper, as our primary interest is in the relationship between designed appearance and perceived robot personality. What is of interest to us, however, is the impact of participant personality traits on participant preferences and behavior in HRI situations, which we will discuss below.

\section{C.Individual Differences in HRI Situations}

The Uncanny Valley theory and its related body of research, suggests universality in human responses to robot appearance and behavior. However, it has been widely acknowledged that norms for preferred behavior and appearance depend not only on situation and culture, but also on individual differences, like personality, aesthetic preferences and interaction styles (Deaux et al., 1993). In light of this, HRI researchers have been studying the role of participant personality and different issues in human-robot interactions. Previous studies have shown that there is a relationship between participant personality and proxemics in HRI situations. Proxemics, or the study of interpersonal distances and personal space (Hall (1966)) during interaction, has been of particular interest to us due to its direct implications for robot 
navigation and motion planning. Walters et al. (2005c) found that participants scoring high in 'Proactiveness' (a personality factor correlating with dimensions of both Extroversion and Psychoticism in the Eysenk Personality Inventory (EPI) personality model), did not allow the robot to come to as close a distance as participants with lower scores in this trait(While this result may seem counter-intuitive, it is likely that the dominance aspects of those two traits may have caused this result). Syrdal et al. (2006) and Gockley \& Mataric' (2006) both found a proxemic tolerance effect for extroversion, in which extroverts tended to tolerate the robot presence to larger extent than introverts. Most research has interpreted these results in terms of Hall's proxemic social spatial theory. However, the differences in measured distances observed for all these human-robot proxemic studies is typically of the order of less than $20 \mathrm{cms}$. Hall's social zone theory was proposed originally to account for broad cultural differences in human-human proxemic distances and the distances were defined in terms of the interactants' arm reach. In most observational studies the actual proxemic distances were estimated visually by experimenters. The social spatial zone distances which are often quoted in relation to human-robot proxemics, are later quantifications of Hall's original estimates (cf. Lambert (2004)). In human-human proxemics it has been found that there are a number of other factors which can affect proximity including fear or uncertainty, the relative status of interactants, physical situation and context, and perceptions of inconsistency (cf. Stratton et al. (1973), Burgoon \& Jones (1976) \& Gillespie \& Leffler (1983)). In recent work we have have addressed some of these aspects of human-robot proxemic behaviour more specifically (cf. Walters et al. (2008)).

Gockley \& Mataric' (2006) also found that extroverts responded better to robots being more 'dominant' in their interactions both in terms of evaluation and task performance. A similar result was found by Tapus and Matarić (2006) that when using differing robot behaviors consistent with human personality types along the extroversion - introversion dimension, participants responded better when interacting with robots whose designed 'personality' matched their own. This result to some extent contradicts the findings of Yan et al. (2004), who found the opposite, namely that introverts rated the extrovert AIBO as more attractive and intelligent than the introvert AIBO, and vice versa. Interestingly, Li et al. (2006) in user studies investigating different verbal interaction styles employed by a robot, shows that users tended to prefer dialog from a domestic robot which exhibited a chattier 'extrovert' conversational style, as opposed to an 'introvert' taciturn style, suggesting that robots that are perceived as more extrovert, may be more acceptable overall to their users. A similar result has been shown by Bickmore and Cassell (2005) in interaction with a Virtual Agent.

\section{D.Research questions}

Related to the above issues, the present study addressed the following main research questions:

1)What is the importance of consistency between robot appearance and behaviors for less human-looking robots?

2)Do people prefer more human-like appearance and behavior in robots that they interact with?

3)Are there any systematic individual differences regarding such preferences?

4)Does personality attribution towards robots follow the pattern of human-human personality attribution at zero acquaintance?

\section{III.METHOD AND PROCEDURE}

The context chosen for the study and associated HRI trials was that of a domestic robot attracting a human's attention using a combination of visual and audible cues. Previously, studies of this type have employed live human-robot experiments in which humans and real robots typically interact in various experimentally controlled scenarios (cf. Walters et al. (2005) \& Walters et al. (2005a)). These live HRI trials are generally complicated and expensive to run and typically test a relatively small sample of possible users. The video methodology chosen was adapted from that employed in previous work. In these studies, the results obtained from participants who viewed a video recording of another person participating in interactions with a robot were found to be comparable to those obtained from participants in live interactions. For more details on this methodology see Woods et al. (2006) and Woods et al. (2006a), but a brief summary of the two relevant comparatory HRI studies is provided here:

Video and Live HRI Trial Pilot Study - The first set of live and video based comparative HRI trials was a pilot study that performed a limited exploratory investigation to assess the potential for the comparability of people's perceptions from live and video HRI trials. Participants took part in live HRI trials and videotaped HRI trials in which the scenario for both trials was identical, involving a robot fetching an object and carrying it to them using different approach directions. Findings from the pilot trials indicated moderate to high levels of agreement for participants' preferences, and opinions for both the live and video based HRI trials. In order to verify these pilot trial findings, and to extend the investigation, a series of video and live HRI trials were performed with a larger sample set and a wider range of HRI situations involving a robot approaching human participants.

Confirmatory Video and Live HRI Trials - In this main study, additional controlled conditions included the human 
participants sitting in an open space, sitting at a table, standing in an open space and standing against a wall. The subjects experienced the robot approaching from various directions for each of these contexts in HRI trials that were both live and video-based. There was a high degree of agreement between the results obtained from both the live and video based trials using the same scenarios. The main findings from both types of trial methodology were: Humans strongly did not like a direct frontal approach by a robot, especially while sitting (even at a table) or while standing with their back to a wall. An approach from the front left or front right was preferred. When standing in an open space a frontal approach was more acceptable and although a rear approach was not usually most preferred, it was generally acceptable to subjects if physically more convenient. Surprisingly, significant comparable results were obtained for both sets of trials with regard to robot approach speed and distance.

Overall, the findings from these experiments supported the use of the video based HRI methodology for developing and trying out new innovative studies that are in the pilot phase of testing. Naturally, there were numerous limitations of using video footage for HRI studies, and it was appreciated that they would not be a replacement for live HRI studies. It was expected that the more interaction is involved between robot and participant in a given trial, the less suitable video trials would be due to the increased importance of aspects of embodiment, dynamics and contingency of interaction. However, for the particular research questions that we considered in this current study, the contingency of robot and human movements plays a less crucial role and therefore the results justify our choice of video-based HRI trials. Applied to the present study, the method consisted of creating three video recordings which were edited to provide a video movie of exactly the same scenario, but each using a robot with a different appearance.

The working definitions of robot appearance for Mechanoid and Humanoid robots used in this article are based on the definitions for animated agents' appearances adopted by Gong \& Nass (2007), and for Android robots from MacDorman \& Ishiguro (2006):

Mechanoid - a robot which is relatively machine-like in appearance. In the HRI trials described here, a robot described as mechanoid will have no overtly human-like features.

Humanoid - a robot which is not realistically human-like in appearance and is readily perceived as a robot by human interactants. However, it will possess some human-like features, which are usually stylised, simplified or cartoon-like versions of the human equivalents, including some or all of the following: a head, facial features, eyes, ears, eyebrows, arms, hands, legs. It may have wheels for locomotion or use legs for walking.

Android - a robot which exhibits appearance (and behavior) which is as close to a real human appearance as technically possible. The eventual aim is to create a robot which is perceived as fully human by humans, though the best that can be achieved currently is for a few seconds under carefully staged circumstances.

Survey results from Khan (1998), Scopelliti et al. (2004) and Dautenhahn et al. (2005) indicated that people do not want domestic robots to exhibit very realistic human-like appearances, so the robot appearances considered for this study were limited to mechanoid or humanoid.

\section{A.The Trial Robots and Videos}

The three robots (Figure 2) were designed by the research team, based on commercially available Peoplebot ${ }^{\text {tm }}$ platforms. Prior to the HRI trials the robots' static appearances (from photographs) were rated on an appearance scale by a panel comprised of 26 researchers from various disciplines including physics, computer science, astronomy and various administrative staff at the University. Figure 3 shows the mean ratings for each robot, the corresponding standard errors and the $95 \%$ confidence interval bands. The scale ranged from very mechanical-looking (1) to very human-looking (20). A Friedman non-parametric ANOVA rated the results as highly significant (Chi Sqr. $(\mathrm{N}=27, \mathrm{df}=2)=44.78431 \mathrm{p}<10^{-6}$ ). In most cases, the ranking order of the robots was the same and the three robots were labeled according to their mean rate values for static appearance: Mechanoid $($ mean $=3.67)$, Basic $($ mean $=6.63)$ and Humanoid $($ mean $=12.22)$. Note that these names are simply used as labels to distinguish the three robots from each other, as none actually looked particularly human-like in appearance. The robots' static appearance (as judged from still photographs) is not the same as the robots' appearance experienced by the participants in the video based HRI trial. The robots in the trial videos were moving and the perceived robot appearance could therefore be considered to be dynamic appearance (that is, also including the behavior of the robot). Thus, dynamic appearance rating is effectively an assessment of the robot as a whole; including not just the robot's static appearance but also includes any movements or other robot behaviors and expressions observed. 

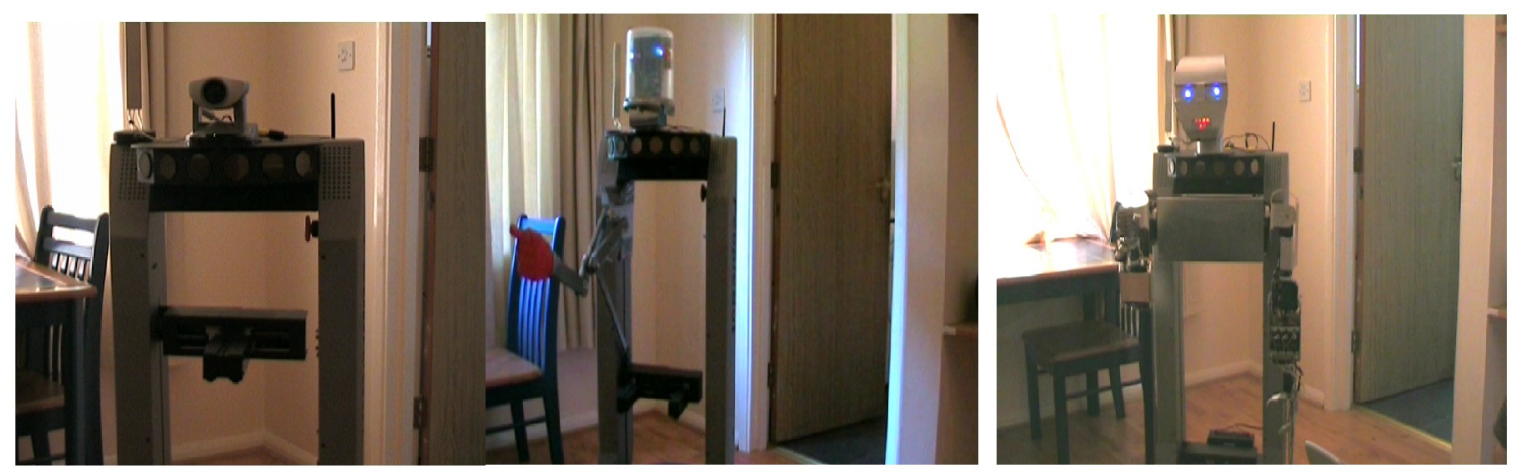

Figure 2. The three robots used for the video based trials

For creating the videos of the three scenarios, each robot displayed a repertoire of attention-seeking cues and behaviors corresponding to their respective robot features. The robots were remotely operated using the Wizard of Oz (WOZ) method, which is widely used in HRI studies. Three different attention-seeking mechanisms were used: manipulator movement, lights, and sound. The manipulators differed between the three robots: The Mechanical-looking robot was fitted with a simple one Degree of Freedom (DoF) gripper which was able to move up or down only. The Basic robot had a simple (one DoF) arm fitted with a compound movement which allowed the robot to lift the arm and make a pointing gesture. The Humanoid robot was fitted with two arms each of seven DoF and was able to make a more human-like waving gesture. Note that it is impossible for either the lifting or pointing arms to make a waving gesture, and conversely, the human-like arms could not easily make a simple lifting or pointing gesture comparable to the actuators of the two other robots.

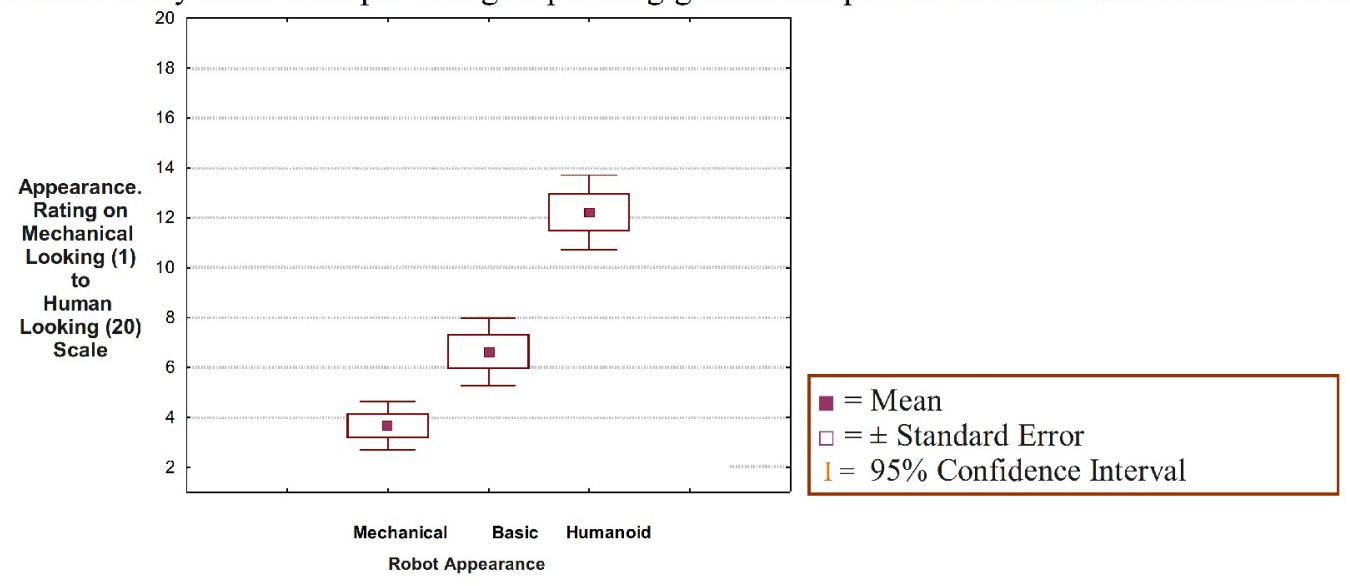

Figure 3. Panel ratings of robot static appearances on the mechanical-to-human appearance scale.

In addition to the movement of the manipulator, visual cues were also used as attention-attracting mechanisms: The Mechanical-looking robot was equipped with a pan and tilt camera unit, fitted with a single flashing light. The Basic robot had a simple head with two flashing lights in place of eyes, and the Humanoid robot had multiple flashing lights in the place of mouth and eyes. Each robot also provided a sound. In the case of the Mechanical-looking robot, a series of two beeps was used. The Basic robot used a poor quality synthesized voice. A high quality recorded human voice was used for the Humanoid Robot. For both synthesized and human voice, the speech content was identical and consisted of the phrase "There is someone at the door." These various attributes to be tested for each of the three robots were therefore categorized as: (dynamic) appearance, gesture, light signal, and sound signal.

Typically, when carrying out a study of this type the various features involved (in this case appearance, sounds, flashing lights and manipulator gestures) would be isolated into a number of separate conditions and a series of tests performed with the various permutations of conditions in order to achieve statistically valid results. However, it is not possible to perform this type of study using robots since the various features of a robot (e.g. appearance, manipulator type, head type, speech or sounds etc.) cannot be isolated from each other. For example, only a robot with a human-like arm will physically be able to perform human-like gestures. Or, if a robot with 'humanoid appearance' speaks with a mechanical voice then it violates the consistency of appearance and behaviour: it will no longer be the 'humanoid' robot that people are judging, but 'something else'. Thus, each particular robot (e.g. humanoid-looking or mechanical-looking) has an overall dynamic appearance which is different from the sum of its individual parts. If any one part or behaviour is changed, effectively this will create a different robot. Likewise, examining individual robot component parts and behaviours in isolation (even in cases where this 
were possible, e.g. isolating speech from the physical robot appearance) means that the concept of a 'robot' would be lost. It is therefore not advisable to consider any one aspect of a robot (such as a particular gesture, speech quality, sound or any other parts or behaviour) in isolation from the rest of the component parts and behaviours which together make up the complete robot. For these reasons, in our study, the appearance and (attention-seeking) behaviour of the robots could not be studied independently in different conditions due to the embodied nature of the robots. This 'holistic' nature of dynamic robot appearance does not allow a clear decomposition of different robot appearance and behaviour features, an approach actually required to perform valid statistical analyses on the different independent features. This exemplifies one of the many methodological challenges that human-robot interaction researchers are faced with. In this paper we present one approach towards the analysis of such data.

\section{B.Personality Assessments.}

The personality model we used in this study was the Big Five Model, measured using the Big Five Domain Scale from the International Personality Item Pool (IPIP) (Goldberg, 1999). This model is used extensively in psychological research, which means that results obtained through the use of this model can be compared easily to those of other psychological studies. The Big Five model assumes five basic personality factors (See Table 1 for a brief description of correlates for the different factors).

TABLE. 1.BIG FIVE PERSONALITY FACTORS (ADAPTED FROM GOLDBERG(1999))

\begin{tabular}{ll}
\hline Emotional Stability & \multicolumn{1}{c}{$\begin{array}{l}\text { Anxiety, depression, self- } \\
\text { consciousness, impulsiveness, } \\
\text { vulnerability }\end{array}$} \\
Extroversion & $\begin{array}{l}\text { Warmth, gregariousness, assertiveness, activity, } \\
\text { excitement seeking, positive emotions } \\
\text { Trust, straightforwardness, altruism, compliance, } \\
\text { modesty, tender-mindedness } \\
\text { Competence, order, dutifulness, achievement } \\
\text { Conscientiousness }\end{array}$ \\
striving, self-discipline, deliberation \\
Imagination, ideas, abstract thought, range of \\
interests
\end{tabular}

Table 2 presents sample items from the IPIP Big Five Domain Scale for both positively and negatively scored items for this personality scale.

TABLE. 2. SAMPLE ITEMS FROM THE BIG FIVE DOMAIN SCALE

\begin{tabular}{ll}
\hline \hline Emotional Stability & I am relaxed most of the time. \\
I get stressed easily. \\
I am the life of the party. \\
I am quiet around strangers. \\
Agreeableness & I sympathize with others' feelings. \\
& I feel little concern for others. \\
Conscientiousness & I am always prepared. \\
& I leave my belongings around. \\
Intellect & I use difficult words. \\
& I am not interested in abstract ideas. \\
\hline \hline
\end{tabular}

\section{C.Scenario}

At the beginning of each trial an introductory video was shown to the participants that included background information about the work of the research group, the purpose of the current trial and detailed instructions for participating in the experiment. As these instructions were recorded, consistency in administering the tests was enhanced. An experiment supervisor was on hand to answer any further questions and to repeat the instructions if necessary. After the introductory video was played, the main trial videos were shown to the participants. 


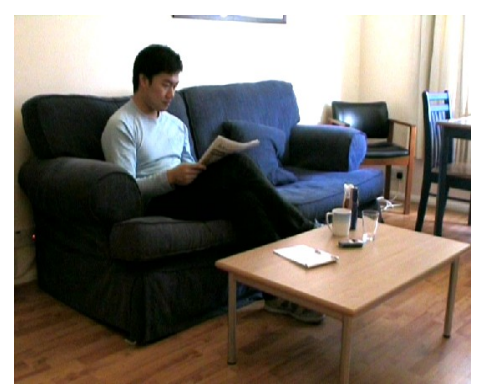

a)
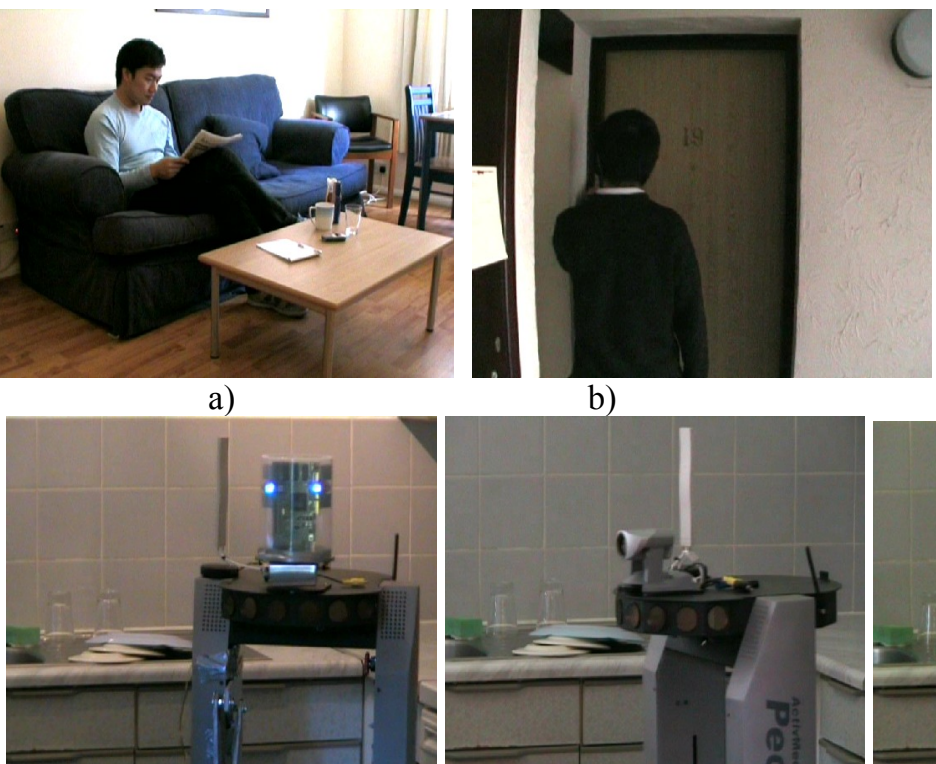

b)
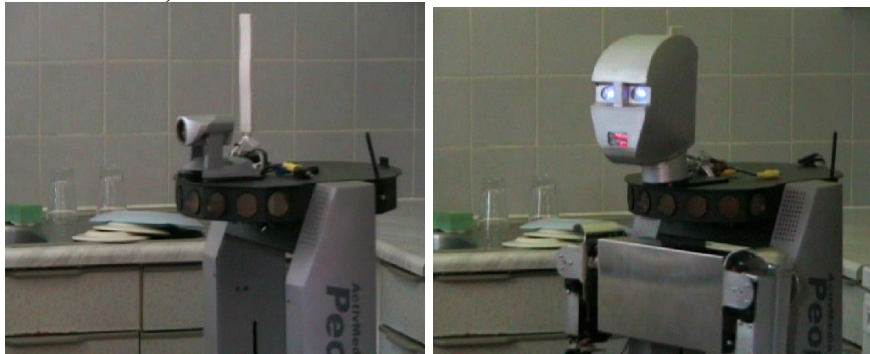

c)
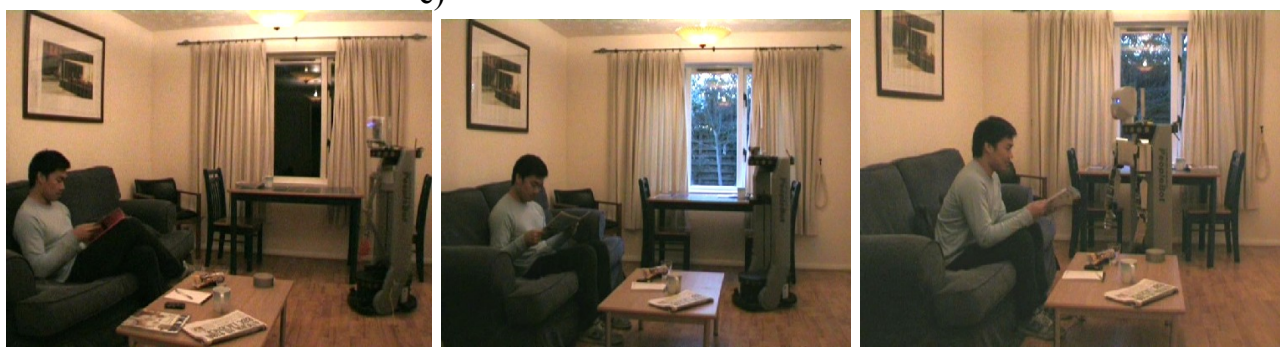

d)
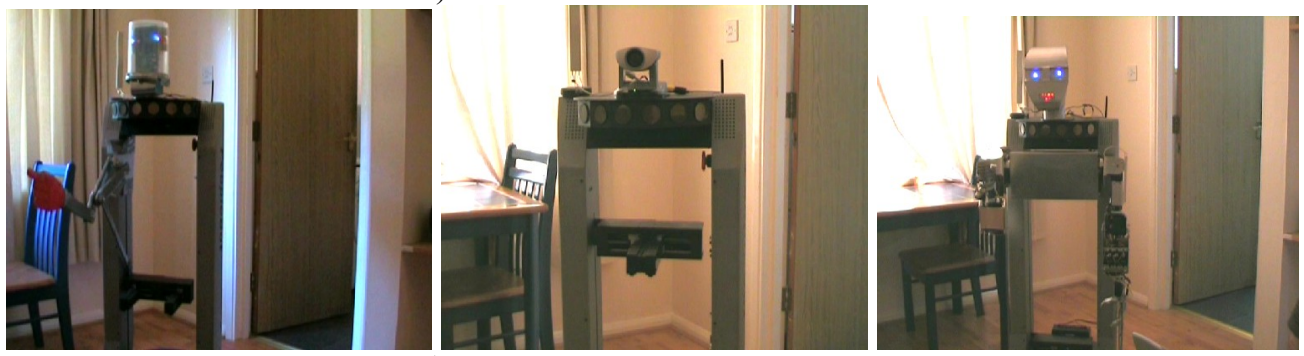

e)
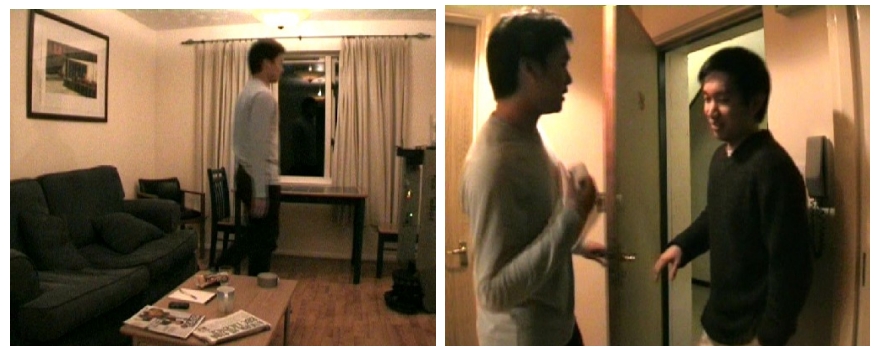

f)

Figure 4. The sequence of events in the trial video. Still photographs captured from the video based HRI trial videos. A person relaxes and listens to loud music (a) when a visitor rings the doorbell (b). The robot hears the doorbell (c) and alerts the person that there is a visitor at the door (d) (e). The person greets the visitor $(f)$. 
The trial videos followed the same scenario which consisted of the following sequence of scenes:

1) A person is shown who is relaxing on a sofa in the living room and listening to loud music (Figure 4a).

2) A visitor approaches the front door and rings the doorbell (Figure $4 \mathrm{~b}$ ).

3) The robot (Mechanical, Basic or Humanoid for each of the three videos) responds to the doorbell, and then acts as if it had assumed that the human has not heard it (Figure 4c).

4) The robot enters the living room and approaches the human. This part of the scenario was shown as viewed from the position of a third party (Figure 4d).

5) The video then switches to the viewpoint of the human (on the sofa), looking directly at the robot. The robot then performs its respective attention-seeking behaviors to indicate that a human response is required: light signal, gesture and sound signal (Figure 4e).

6) The human is then seen following the robot out of the room, and then opening the door and greeting his visitor (Figure 4f).

The trial videos were recorded in the University of Hertfordshire Robot House, a naturalistic home environment for Human-Robot interaction trials which is more ecologically valid than a simulated domestic setting in a converted or adapted university lab environment that we have used in previous trials, cf. (Syrdal et al., 2006), (Woods et al., 2006).

\section{D.Participants and Procedure}

The three videos were shown to a total of 79 undergraduate students, in three separate group sessions ranging in size from $20-30$ individuals at a time. The participants were mainly students $(98 \%)$, only $8 \%$ were females. A majority $(82 \%)$ said they had some familiarity with entertainment type robots and $5 \%$ of the participants were left handed. The participants completed the questionnaires individually. Generally, in order to reduce social facilitation effects (Woods et al. 2005), the group sessions did not involve any discussion of the main trial videos and how participants rated the different robots. The participants signed consent forms, provided basic demographic details including, background, gender, handedness and age, as well as computer and programming experience before they were exposed to the introductory video. They were then shown the three main trial videos, each group in a different order, of a robot attracting attention from a person - featuring the Mechanical, Basic and Humanoid robots. After the three videos were displayed, a slide showing the three robots (Figure 2) with their names and features was projected on the main screen as an aid to participants' memory as to the identity of the robots in the videos. The participants were then asked to fill in a questionnaire in order to collect their opinions and preferences towards the three robots and the various attention-seeking behaviors. Details of the relevant questions from the questionnaire are provided below in the Results and Analyses section. For each session, the three robot scenario videos were presented in a different order. As there were only three group video sessions, not all possible permutations of video presentation order could be covered.

\section{IV.TRIAL RESULTS AND ANALYSIS}

\section{A.Robot Appearance Ratings}

For reasons discussed previously, it was not possible to fully isolate and cross combine the various appearance and attention- seeking behaviors, as the robot features tested were not truly independent. For analysis purposes, it was assumed that dynamic robot appearance would be closest to an independent variable. The other attention-seeking behaviors would then be perceived by the human test participants as either being consistent or inconsistent with the overall dynamic appearance of each robot. To measure this, each participant provided a set of ratings on a Likert scale $(1=$ Dislike a Lot, 3 $=$ Neutral, $5=$ Like a Lot) for their preference for each robot's (dynamic) appearance, light signal, sound signal and gesture behavior. For example the Mechanical-looking robot exhibited a single flashing light, a beep sound and a simple lifting gripper gesture. Participants rated their preference for dynamic appearance and these three attention-seeking behaviors for the Mechanical robot. In the same way the preference ratings for the twin flashing lights, the low quality synthesized voice and the pointing arm gesture were obtained for the Basic robot. The multiple flashing eye and mouth lights, the high quality (recorded) human voice and the waving arm gesture were likewise rated for the Humanoid robot.

Friedman non-parametric ANOVA for repeated measurements were performed on all the participant's ratings. Highly significant differences were found for the dynamic appearance scores (Chi Sqr $=33.10425, \mathrm{~N}=76, \mathrm{DoF}=2, \mathrm{p}<10^{-6}$ ). The mean results are illustrated below (Figure 5), along with a visual indication of standard error and 95\% confidence interval bands. In general, the participants' ratings of robot dynamic appearance indicated that overall they preferred the Humanoid robot, followed by the Basic robot and finally the Mechanical-looking robot. 


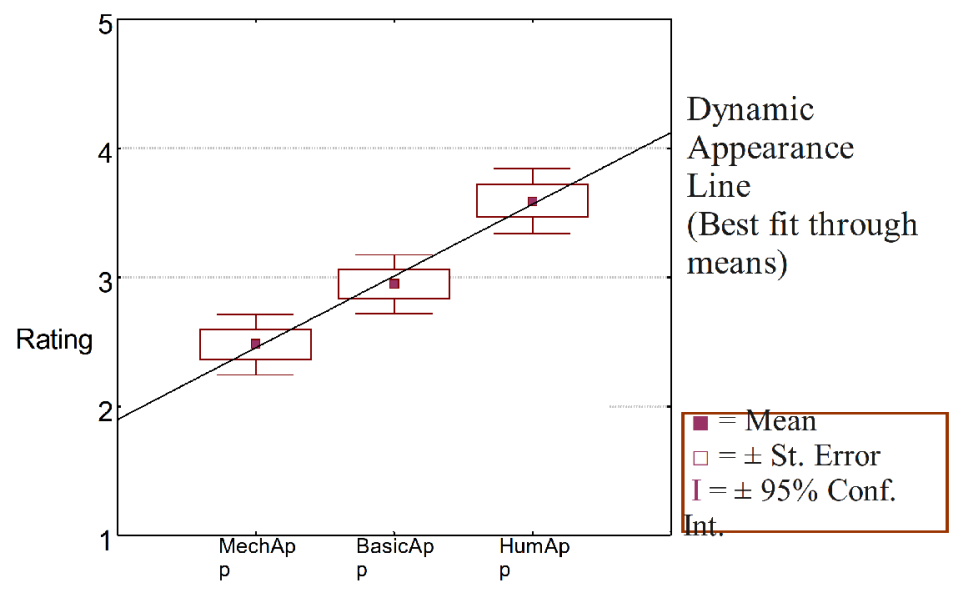

Figure 5. Participants' mean appearance ratings for the three robots. Appearance Preference Ratings. $(1=$ Dislike a lot, $0=$ Neutral, $5=$ Like a lot. $)$.

\section{B.Robot Attention-Seeking Behaviors}

The three sets of attention-seeking behavior employed by the three robots were not truly independent from each other, or from the respective robots' appearances. However, as argued previously, the different dynamic appearances of the three robots can be considered to encapsulate the main overall impression of an individual robot by each trial participant. We therefore used the robot's (dynamic) appearance rating as a base line for gauging the contribution of each of the individual attention-seeking behaviors. For this purpose the line marking the best linear fit of the mean appearance preference ratings was drawn (see Figure 5). (Note that this line only acts as a visual guide to allow easy comparison with the other attentionseeking behaviors. Because the order of the three robot types along the horizontal axis is at most ordinal, no conclusions should be drawn about the shape of this line per se.)

It can be seen that when compared to the means obtained from the overall appearance ratings, the Humanoid robot's waving gesture is rated similarly to the same mean value as dynamic appearance. For the other two robots, the mean for the lifting gripper gesture is rated better than the overall Mechanical robot appearance rating, and the pointing gesture is rated less then the Basic robot appearance rating (Figure 6). The differences in rating between the gestures of the three robot types were highly significant by the Friedman test (Chi Sqr $\left.=25.73799, \mathrm{~N}=76, \mathrm{df}=2, \mathrm{p}<.10^{-6}\right)$.

Friedman tests showed that the differences between the participants' ratings of the light signals and sound signal were both highly significant: For the light signals $\left(\mathrm{Chi} \mathrm{Sqr}=.25 .74, \mathrm{~N}=76\right.$, df $=2, \mathrm{p}<10^{-6}$ ), the single light of the Mechanical robot and the two lights of the Basic robot were more liked than their respective overall appearance ratings. The multiple flashing lights on the Humanoid robot, however, were rated as less liked than the overall dynamic appearance rating might suggest (Figure 8). The ratings for the sound signals (Chi Sqr $=62.86, \mathrm{~N}=77, \mathrm{df}=2, \mathrm{p}<10^{-6}$ ) indicated that the simple beeps of the Mechanical robot were disliked more than the corresponding Mechanical robot's dynamic appearance. However, for both robots which used speech, this was actually liked better than their respective dynamic appearance ratings (Figure 7). 


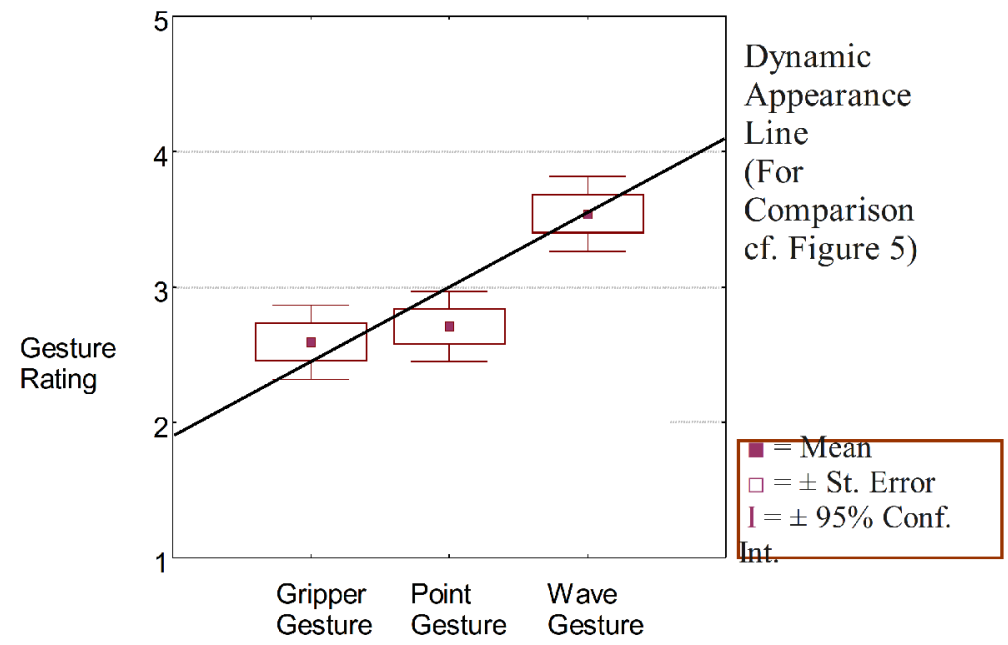

Figure 6. Ratings of the robots' gestures. (Ratings; $1=$ Dislike a lot, $0=$ Neutral, $5=$ Like a lot).

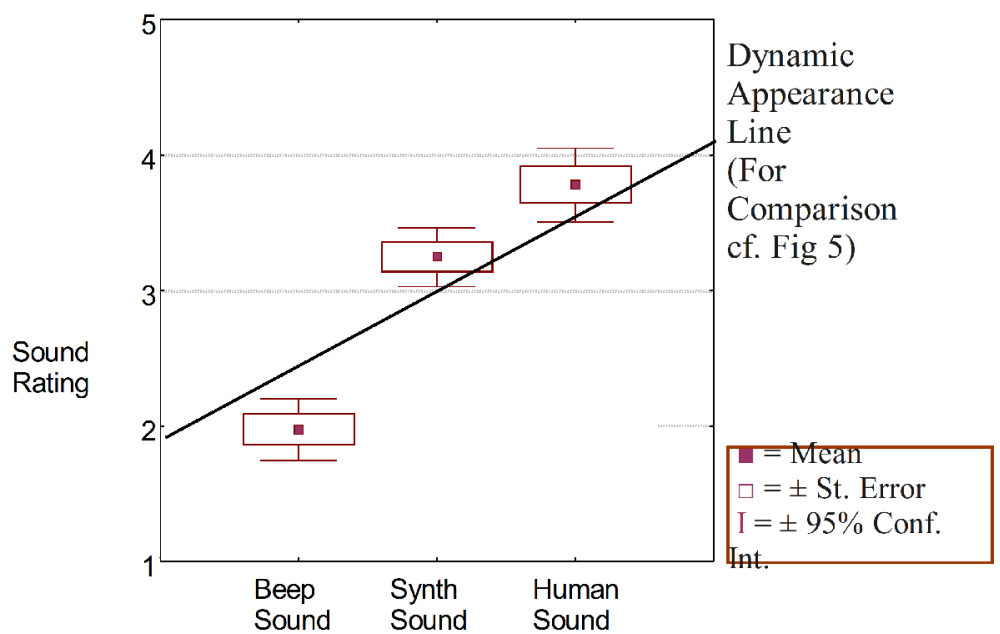

Figure 7. Ratings of the robots' gestures. (Ratings; $1=$ Dislike a lot, $0=$ Neutral, $5=$ Like a lot).

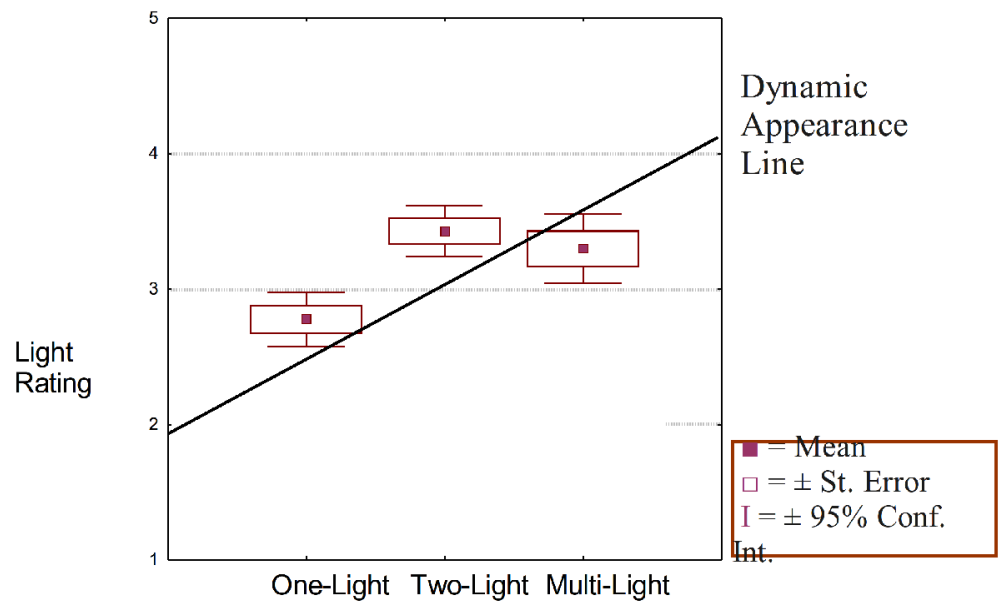

Figure 8. Ratings of the robots' light signals. (Ratings; $1=$ Dislike a lot, $0=$ Neutral, $5=$ Like a lot). 


\section{C.Personality and Appearance Ratings}

In order to find any systematic effects of participant personality on appearance preferences, Spearman non-parametric correlations were run between the preferences indicated for each robot appearance and the personality traits. A significant correlation was found between Extroversion and Mechanical Appearance preferences $(\rho=-.263, p=.022)$ and Emotional Stability and Mechanical Appearance preferences $(\rho=-.313, p=.007)$. These two correlations suggest that participants scoring low in Extroversion prefer the Mechanical appearance to a greater degree than other participants.

\section{D.Perceived Robot Personality}

Perceived robot personality was measured in the questionnaire using the following items which were rated by the participants along a 5-point Likert scale ranging from 1 (Not at all) to 5 (Very). Note that a general intelligence item was substituted for the 'Intellect/Openness to Experience' dimension, see Table 3.

\section{TABLE. 3.ROBOT PERSONALITY ITEMS}

\begin{tabular}{ll}
\hline Emotional Stability & $\begin{array}{l}\text { How relaxed and content, or stressed and } \\
\text { easily upset was the robot? } \\
\text { Extroversion }\end{array}$ \\
Hgreeableness & $\begin{array}{l}\text { How interested/disinterested in people was } \\
\text { the robot? }\end{array}$ \\
Conscientiousness & $\begin{array}{l}\text { How organized and committed or } \\
\text { disorganized/uncommitted was the robot? } \\
\text { how intelligent or unintelligent was the } \\
\text { robot during its tasks? }\end{array}$ \\
\hline
\end{tabular}

The mean assigned scores for the different personality traits according to appearance can be found in Table 4 and Figure 9 .

TABLE. 4.MEAN SCORES FOR PERCEIVED ROBOT TRAITS

\begin{tabular}{llll}
\hline \hline Personality Trait & $\begin{array}{l}\text { Mechanical } \\
\text { Robot }\end{array}$ & $\begin{array}{l}\text { Basic } \\
\text { Robot }\end{array}$ & $\begin{array}{l}\text { Humanoid } \\
\text { Robot }\end{array}$ \\
\hline Emotional Stability & 3.22 & 3.33 & 3.57 \\
Extroversion & 2.35 & 3.08 & 3.72 \\
Agreeableness & 2.47 & 3.22 & 3.64 \\
Conscientiousness & 3.23 & 3.45 & 3.75 \\
Intellect & 2.89 & 3.24 & 3.67 \\
\hline \hline
\end{tabular}



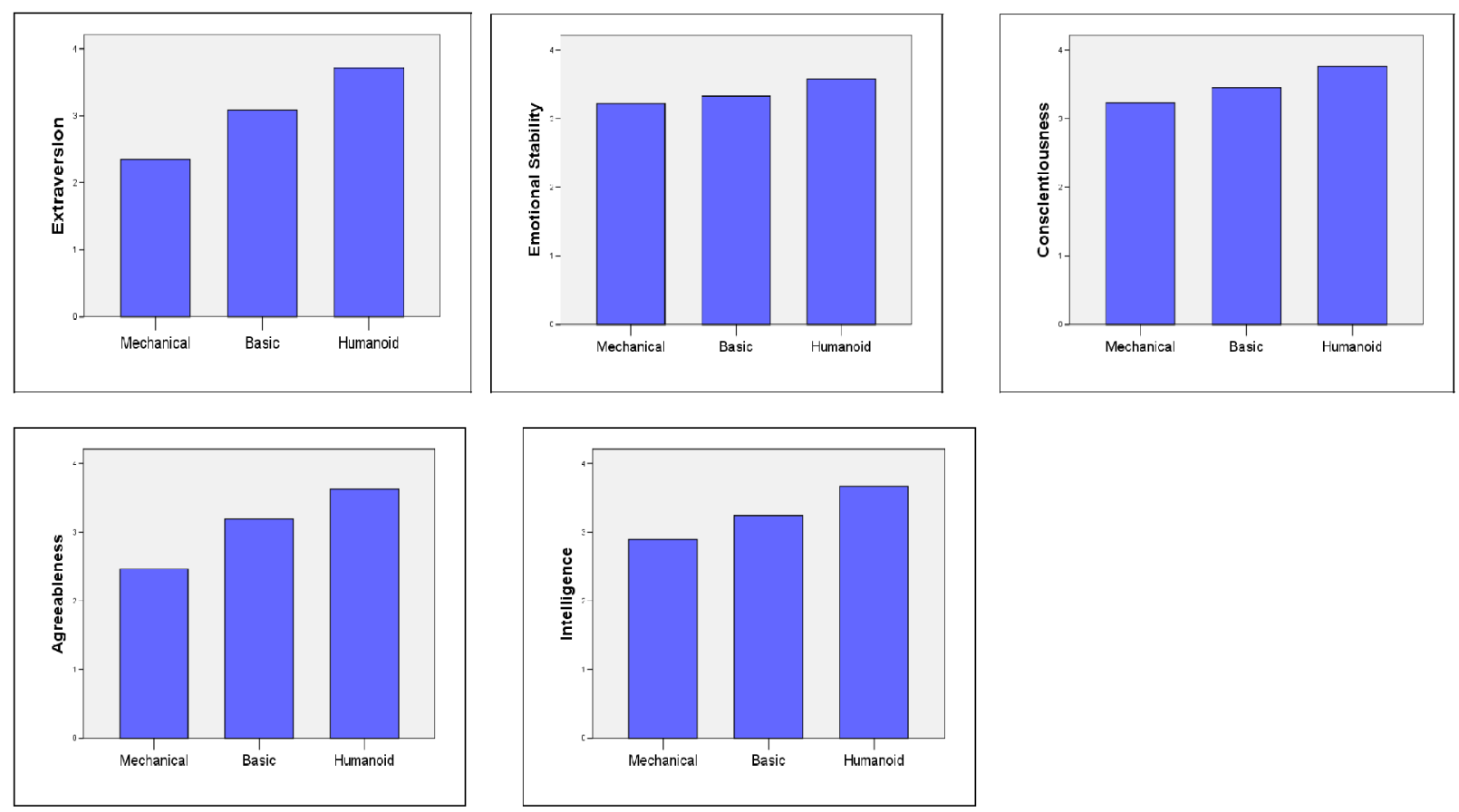

Figure 9. Mean scores for perceived robot traits

Results suggest that for all the traits, the Mechanical robot scored the lowest overall, followed by the Basic and then the Humanoid robot. The relationships between the perceived robots personality traits and (dynamic) robot appearances were assessed by running a series of ANOVAs:

1. For Extroversion there was a significant main effect for robot appearance $(F(2,75)=51.62, p<0.001)$. Significant differences for Extroversion were also found between all three robot appearances $(p<0.005)$.

2. For Agreeableness there was a significant main effect for appearance $(F(2,75)=37.80, p<0.001)$. Significant differences for Agreeableness were also found between all three robot appearances $(\mathrm{p}<0.005)$.

3. For Conscientiousness there was a significant main effect for appearance $(F(2,75)=9.855, p<0.001)$. Significant differences for Conscientiousness were also found between the Humanoid appearance and the two other appearances $(\mathrm{p}<0.005)$.

4. For Emotional Stability there was a significant main effect for appearance $(F(2,75)=4.014, p<0.05)$. No significant differences were found for Emotional Stability between the conditions.

5. For Intellect there was a significant main effect for appearance $(F(2,75)=21.87, p<0.001)$. There were significant differences for Intellect between all three appearances $(\mathrm{p}<0.05)$.

These results suggest that there were differences between the magnitude of difference between the traits, with Agreeableness, Extraversion and Intellect being the traits with the clearest difference between the robot appearances, while the differences were smaller for Conscientiousness and Emotional Stability.

\section{E.Robot Personality as a Uni-Dimensional Construct.}

Due to the trends apparent for all the traits, the possibility of these traits being uni-dimensional scales (i.e. measuring one underlying construct) for each robot appearance was tested using Cronbach's Alpha. The results of this analysis can be found in table 5 below and support the notion of perceived robot personality as a uni-dimensional construct of data in this investigation, see Figure 10.

\section{TABLE. 5. INTER-ITEM RELIABILITY FOR ROBOT PERSONALITY TRAITS}

\begin{tabular}{|l|l|}
\hline Robot Type & Cronbach's Alpha \\
\hline Mechanical & .74 \\
\hline Basic & .68 \\
\hline Humanoid & .80 \\
\hline
\end{tabular}

1) Mechanical Appearance:

The model explained $55 \%$ of the variance and was significant $(\mathrm{F}(3,74)=25.57, \mathrm{p}<0.001)$. The predictors were 
mechanical robot appearance preferences $(\beta=0.612, \mathrm{t}=7.13, \mathrm{p}<0.001)$, Extroversion $(\beta=.235, \mathrm{t}=2.75, \mathrm{p}<0.01)$, and knowledge of computer programming $(\beta=-0.367, \mathrm{t}=-4.34, \mathrm{p}<0.001)$. This suggests that the more participants liked the Mechanical robot appearance, and the more extrovert they were, the more likely they were to rate the robot higher in the personality traits. There was a negative relationship between computer programming and perceived robot personality, suggesting that participants that had experience in computer programming were likely to rate the Mechanical appearance lower in the personality traits than non-programmers.

2) Basic Appearance:

The model explained $21 \%$ of the variance and was significant $(\mathrm{F}(2,75)=8.64, \mathrm{p}<0.001)$. The predictors were basic robot appearance $(\beta=0.343, \mathrm{t}=3.14, \mathrm{p}<0.005)$ and Extroversion $(\beta=0.319, \mathrm{t}=2.92, \mathrm{p}=0.005)$. This suggests a similar relationship as for the Mechanical robot appearance. There were no significant negative predictors.

3)Humanoid Appearance:

The model explained $19 \%$ of the variance and was significant $(\mathrm{F}(1,76)=16.34, \mathrm{p}>0.001)$. The only significant predictor was Humanoid robot appearance preferences $(\beta=0.430, t=4.04, p>0.001)$. This suggests that participants preferring this appearance were more likely to rate it higher for the personality traits. Figure 9 below gives an overview of the relationships between the predictors and perceived robot personality.

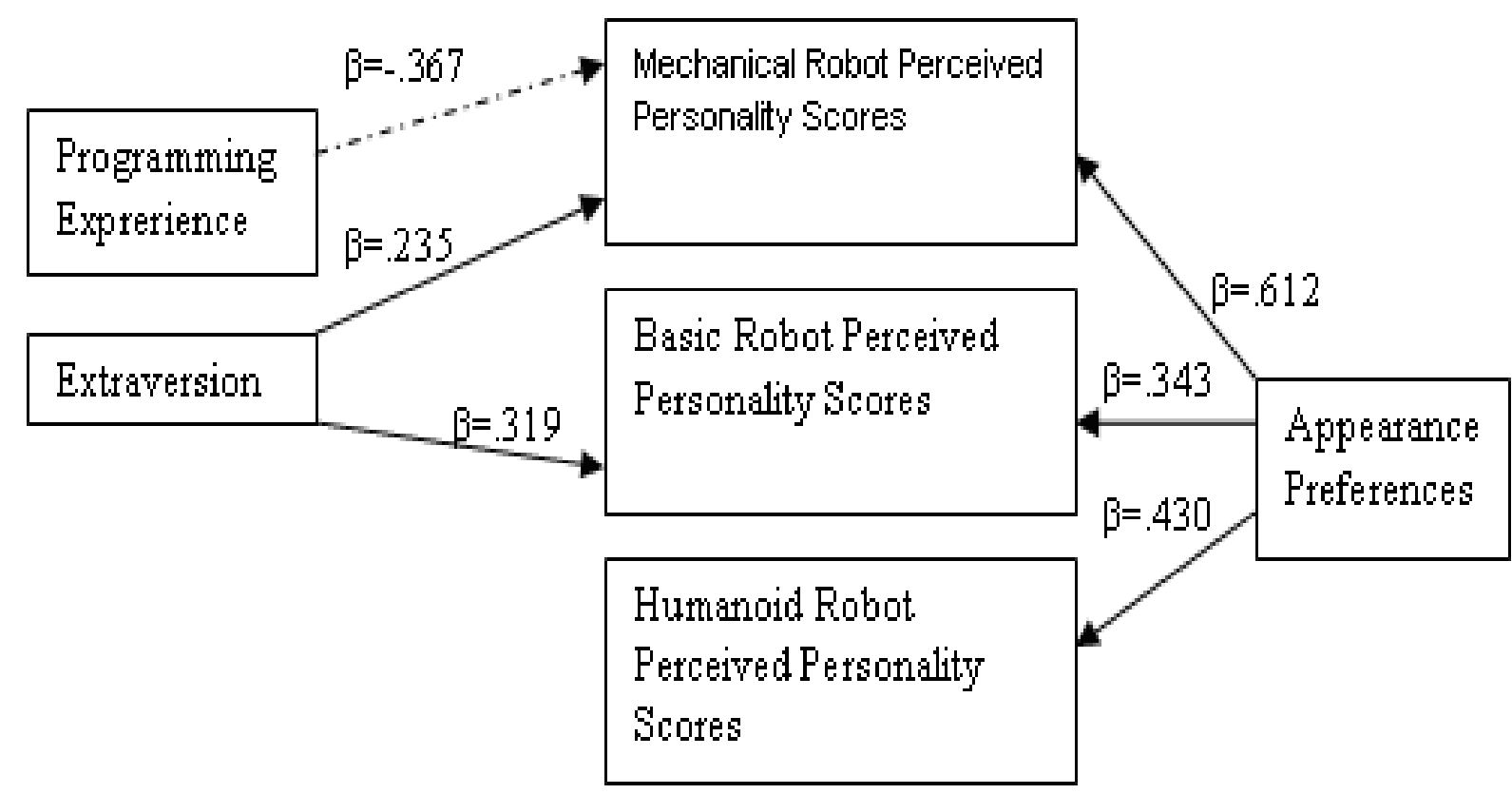

Figure 10. Predictors of perceived robot personality scores (all coefficients significant, $p<.01$ ).

\section{F.Qualitative Analysis of Participant Responses to Questionnaires}

A data driven, qualitative analysis of the participants' reasons for their most preferred robot appearance was conducted in order to categorize these and relate the categories to the different robot appearances. The categories identified from the participants' responses were:

1) Aesthetic - Used to describe statements that referred to the purely aesthetic value of the robot's appearance.

Examples: 'Because it looked more aesthetically pleasing'; 'The appearance of the robot was better'

2) Socio-Emotional Response - Used to describe statements that referred to emotional and/or social responses associated with the particular appearance. Examples: 'It made me feel more comfortable'; 'less scary than the others'. 
3) Functional/Task-related - Used to describe statements that referred to the suitability of a certain appearance to various tasks, as well as the usefulness/saliency of its gestures and voice-types. Examples: '...performed faster than the other two'; 'Voice was informative'.

4) Truthful Representation - Used to describe statements that referred to whether or not the robot's appearance gave a truthful representation as to its nature and capabilities as a home robot. Examples: 'Close to my expectations of a robot in the home'; '...it looked like a robot and that's what it is'; '[The Robot] didn't pretend to be what it wasn't'

5) Anthropomorphic Quality - Used to describe statements that referred to human-like qualities in the robot's appearance. Examples: 'It had some human characteristics'; '...it is closer to human appearance'; 'It is unnecessary to have a human appearance'

For an overview of the proportions of statements that contained each category according to the most preferred robot appearance see Figure 11 and Table 6.
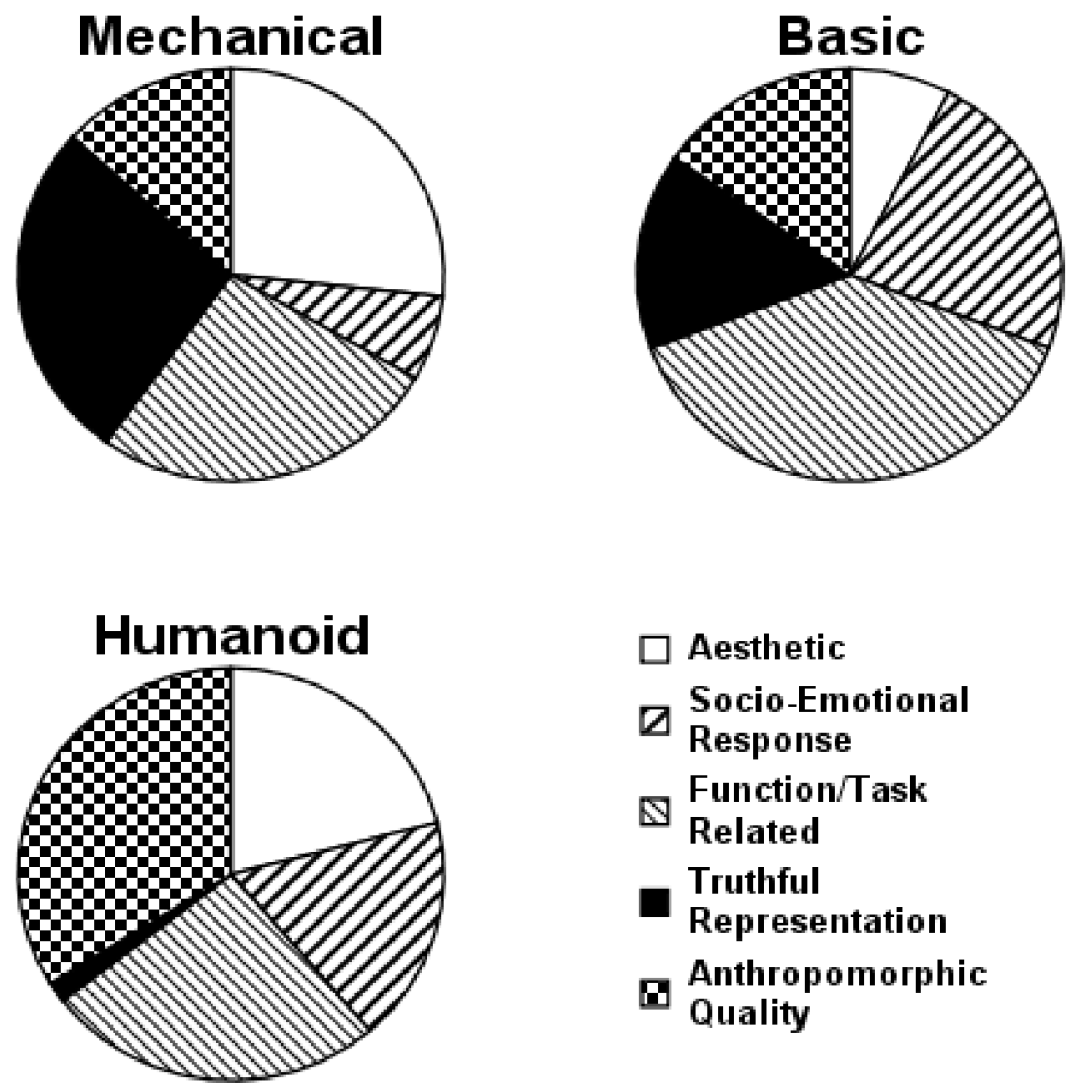

Aesthetic

Socio-Emotional

Response

Function/Task

Related

Truthful

Representation

Anthropomorphic

Figure 11. Proportion of categories according to robot appearance 
TABLE 6. RESULTS OF QUALITATIVE ANALYSIS OF PARTICIPANT RESPONSES

Proportions of categories according to robot appearance

$\%$ within Robot Appearance

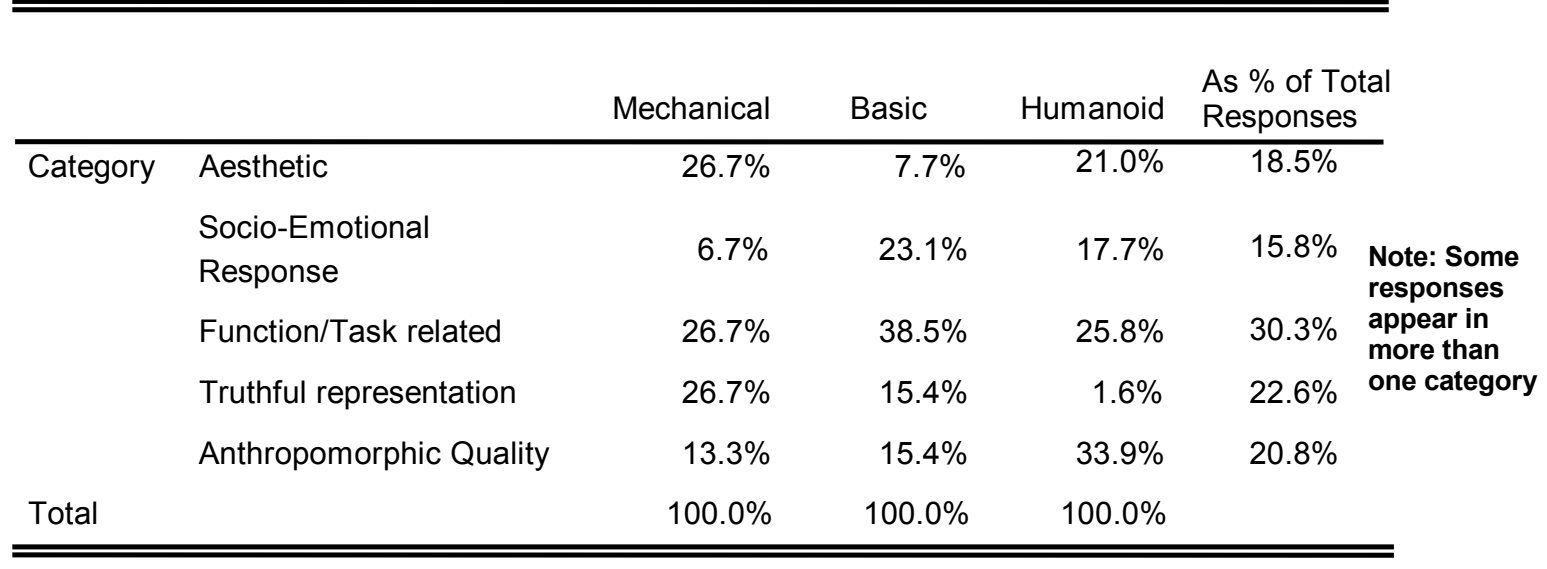

The following results emerged regarding the different categories:

Aesthetic - Statements referring to purely aesthetic aspects of the robot appearance were the most common for the mechanical and humanoid appearance. For the mechanical appearance, the majority of these statements referred to the simplicity of its design. For the humanoid appearance, however, these statements primarily consisted of stating that its appearance was more aesthetically pleasing than the others, without explaining the reasons for this aesthetic value.

Socio-Emotional Response - These statements were mostly prevalent for the Basic and Humanoid appearances. For the Humanoid appearance, these statements tended to focus on how its human-like appearance allowed for a more comfortable and natural interaction - 'I think a humanoid appearance is...easier to adapt to'; 'It made me feel more comfortable and like another human was there'. For the Basic appearance, the responses focused on how this appearance was comfortable and nonthreatening. - '...it doesn't scare children'; '...I would be more comfortable if I was approached by it'.

Function/Task Related - These statements were common for all three robot types. For the Mechanical and Basic robot types, these statements tended to highlight the importance of the robot's capabilities over that of its appearance - 'It was perfect at attracting your attention... face appearance is not important' ; '...no need to look like a human as long as it does what it is supposed to do'. The participants who preferred the Humanoid appearance however, tended to describe how the Humanoid appearance allowed the robot to perform its task more efficiently - 'its motion is very human-like, and so can be understood easily'; 'It clearly helps, so there is no difficulty in understanding'.

Truthful representation - This category was primarily found amongst the participants preferring the Mechanical and Basic appearances, but was not prevalent for the Humanoid appearance. For the Basic and Mechanical appearances, the fact that the robot's appearance had a direct relationship with its nature and capabilities as a robot was seen as an important factor - 'It is not trying to be too realistically human' ; 'Looks more like a [home] appliance'; 'It was the least contrived - it was what it was'.

Anthropomorphic quality - Statements regarding this category were most commonly found for the Humanoid appearance, but did appear for both Mechanical and Basic appearances as well. For the Humanoid appearance, this category appeared on its own, suggesting that, for many of the participants, anthropomorphic appearance in itself was a desirable quality - 'Human-like arm, detailed head' ; 'It is the most similar to humans'. It also appeared, as mentioned earlier, together with task related statements. For Basic and Mechanical appearances, it almost exclusively appeared as described in the Function/Task Related category. 


\section{V.DISCUSSION AND CONCLUSIONS}

\section{A.Appearance Ratings - Feature Consistency}

In all the results above, any Likert value below 3 implies that a feature or behavior was disliked. Any value above 3 indicates that a feature was liked overall. The Basic robot's attributes were all close to the neutral value of 3 , implying that overall it was not particularly liked or disliked. The Mechanical robot's attributes consistently fell into the category below 3 indicating that overall it was mildly disliked. Other interesting observations are that speech, even of poor quality, was liked in contrast to simple beeping sounds, which were disliked. Overall, it can be seen that the Humanoid robot's appearance and behaviors were all liked to some degree. However, the multiple flashing eye and mouth lights features were not liked to the same degree as the rest of the Humanoid robot's attributes and were actually rated as less liked overall than the twin flashing lights on the Basic robot ${ }^{1}$. The left hand side of Mori's original diagram (Figure 1) illustrates his idea that humans are more approving of robots which have more human-like appearance and behavior (up to a certain point). It is interesting here to plot the panel ratings (from Figure 3), which were purely judging robot static appearances (on a mechanical to human like looking scale), against the actual dynamic appearance ratings of the HRI trial participants (Figure 10). In Figure 10 the independent panel's ratings on the mechanical-human appearance scale means (range 1 to 20) were divided by 4 in order to show them on the same scale as those for the trial participant's dynamic appearance ratings. The ratings for the robots, for both static and dynamic appearance, increase from Mechanical-looking to Basic to Humanoid robot (Figure 12), thus providing support for the left hand side of Mori's diagram. The fact that participants tend to rate dynamic appearance higher than static appearance also supports Mori's view that robot behavior is important in shaping humans' views of robots. There are insufficient data points (and it would be questionable anyhow because the dynamic appearance ratings are based on a Likert scale which is only ordinal) to show if the relationship between increasing human-like appearance and human approval is actually linear or some other functional relationship.

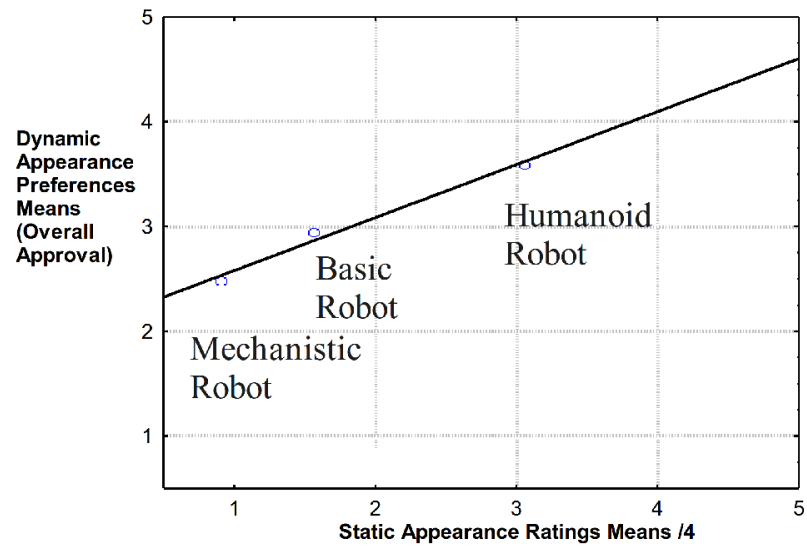

Figure 12. Robot static appearance ratings vs. robot dynamic appearance preferences.

The labeling of the robot types (Mechanical, Basic, and Humanoid) could be open to critique, because such 'leading names' might have influenced the judgments by the subjects. However, the various attributes of each robot were rated separately by participants. Also, the labels were given in order to elicit participants' opinions towards these three fundamentally different categories of robots, and not necessarily restrict their opinions to the three specific designs presented here. That the flashing lights of the 'Humanoid' robot were not actually liked as much as the overall appearance of the robot suggests that participants were not unduly influenced by the names used for the three robots. Future trials could avoid the use of leading names for the robots to be rated by trial participants in order to detect any differences of the results compared to our study.

\section{B.Individual Differences in Appearance Preferences}

Our results do not support the notion that increasing the human-likeness of a robot will necessarily make it more preferable to interact with. Negative correlations were found between appearance preferences for the mechanical appearance and the two personality traits, Extroversion and Emotional Stability, in which participants scoring low on these traits preferred the mechanical appearance to a larger extent than other participants. This would suggest a preference for robots without anthropomorphic features in these participants. This could be seen as a general disposition to prefer a robot companion that does not require interactions that are as social in nature as what would be expected of the more

\footnotetext{
${ }^{1}$ We designed the flashing mouth and eyes as we believed they would attract attention to these more human-like features, but results suggest that these choices were not perceived as consistent with the robot's appearance.
} 
anthropomorphic appearances. The use of speech by the Basic and Humanoid robot may have had a strong impact on this. As introverts and individuals scoring low on Emotional Stability would find social interactions more stressful than other individuals, this could lead to the conclusion that some users may find a less explicitly anthropomorphic means of interacting with a robot companion more suitable than more extrovert and emotionally stable users. These results can also be taken in support of the notion of matching robot personality with that of the user, as the Mechanical appearance was rated the least extrovert of the three robot appearances.

From the participants' supplementary comments about their reasons for robot appearance preferences, which were obtained from the questionnaires, it can be seen that there are five main categories of responses. From the frequencies of questions from these categories, it can be concluded that overall the participants did not have strong views about the Basic robot in terms of appearance, compared to the Mechanical and Humanoid robot. However, the Basic robot appearance was overall assessed as truthful in terms of its capabilities, and was also generally described as nonthreatening and comfortable to interact with. Therefore, although most participants overall preferred the Humanoid robot, some few individuals did strongly prefer the Mechanical robot over the Humanoid robot. It may be inferred from this that a robot appearance which does not exhibit too many human-like attributes may be preferred for interactions in a public arena (e.g. as a museum guide, or a receptionist) as it would provide a robot appearance that would not be most people's first choice, but would be reasonably comfortable and acceptable for the majority of people to interact with.

\section{C.Robot Personality Attribution}

The results of this paper suggest that the processes of assigning personality traits to a robot have similarities with that of assigning the same traits to other humans: Firstly, what we appear to see is an overall 'halo effect' (in which a positive rating on one dimension leads to higher scores on other dimensions, ) where liking for the particular robot appearance led to a higher rating for all its personality traits (which consisted of items that would be seen as good and desirable by most participants). This effect can be considered to account for the overall differences between the robots as the ratings for personality traits corresponded to the overall ratings for robot appearance preferences, and also for the role of appearance preferences as predictors for perceived robot personality. Secondly, the above effect is greatest for Extroversion, Agreeableness and Intellect, while it is weaker for Conscientiousness and Emotional Stability. This does, to some extent correspond with the findings of Kiesler \& Goetz (2002), who found that participants' mental models for robots were richer for the personality trait of Extroversion and less so for Emotional Stability. Also, as the item used to measure Agreeableness was directly related to social behavior, the large effect size for this trait may reflect a similar phenomenon to that proposed by Kiesler and Goetz as well. As our study did not give a 'not applicable' option for the trait ratings (in order to encourage the subjects to make decisions), richness of a mental model would here be indicated in the ability of the participants to differentiate between the robot appearances for a particular personality dimensions, reflected in the variances. It should be noted that the findings also correspond to previous studies on human-human ratings of strangers, in that the differences for Extroversion, Conscientiousness and Agreeableness were greater than that for Emotional Stability. This fits well with the notion of Emotional Stability being the most difficult trait to accurately rate strangers. Combined with the halo effect, this points to a similar mechanism for assigning robot personality as for that for assigning personality to another human being and also accounts for the perceived intelligence of the robot. The impact of the halo effect on perceived intelligence in humans is well documented in the literature and so it is a strong possibility that this effect may also impact on perception of robot intelligence.

With regard to individual differences in the attribution of robot personality, a most interesting result was that Extroversion in participants' personalities was a significant predictor for higher personality ratings for the Mechanical and Basic appearance, which overall had significantly lower personality ratings than the Humanoid appearance. This effect may be the result of a general propensity in extroverts to respond to other objects in a more social manner. As extroverts tend to be more adept at social interactions than introverts, they are also more likely to respond to, and interact with external objects in a social manner. Such an effect was described by Luczak et al. (2003) who found that extroversion was a significant predictor for social interaction with technological artifacts. Our results seem to confirm that extroverts are more likely to adopt anthropomorphic heuristics when interacting with non-human animals and objects.

\section{Conclusions and Future Work}

Our findings have implications for the designers of robots which must interact with humans. Where a robot behavior or feature is rated by humans as less liked or approved of than a robot's overall appearance might suggest, there will inevitably be a degree of disappointment. This may explain why humans become rapidly discontented with toys and robots which have a very interesting and anthropomorphic visual appearance, but prove to be disappointing after actual interaction takes place. The number and range of robots tested in our study is not large enough to provide statistically hard evidence to support the whole of Mori's diagram. Also, none of the robots had an appearance which was human-like enough to trigger the uncanny valley effect, so the results obtained here can only be taken as evidence to support the left hand side of Mori's diagram.

Most participants preferred the humanoid robot appearance overall, except for a few individuals who favored a robot with 
a mechanical appearance. The supplementary responses indicate that a Basic robot appearance may be more acceptable to a majority of the participants. It is also worth noting that there is more to the participant preferences than just aesthetic or purely functional considerations when designing robot appearance. For example, appearance requirements for a tour-guide robot will be different from those of a personal, or domestic, robot. The tour-guide appearance will have to satisfy (i.e. not offend or irritate) a wide range of possible users, whereas the personal robot's appearance can be much more heavily customized to the individual user's preferences and requirements, e.g. for a personalized robot companion (Dautenhahn (2004).

Another implication of these findings is that differences in robot appearance lead to marked differences in perceived robot personality. This findings support those of Goetz et al. (2003) and Tapus and Matarić (2006) and it is therefore reasonable to assume that differences in robot appearance will lead to differences in perceived robot (personality, and as such the) ability to perform tasks in human-centered environments. The issue of individual difference also needs to be addressed. One example of dealing with such an issue might be for robots that overall score high on extroversion due to appearance, compensate for this by adjusting its behavior to be more 'introvert' when dealing with users that prefer a less anthropomorphic form.

The use of video, rather than live interaction might have had a systematic effect on the results. While, as described in Section II, we have shown that for scenarios with little interaction between robot and subjects, video trials have shown to be a valid method compared to live trials, other scenarios involving more interaction in live situations might provide different results. Also, subjects were exposed to the robots only briefly, and as such results from repeated and prolonged interactions may differ from what was shown in this study. We do contend, however that the initial impression and evaluation of a robot companion is important in HRI. In many instances, individuals will interact with robots in situations that are limited in both time and scope, such as in museums, hospitals or if visiting homes that own robot companions. We do recognize the need for longitudinal studies that examine how impressions and evaluations of social robots change over time. For such an undertaking, establishing baselines at zero acquaintance, as this paper does, is important to accurately track these changes.

More longitudinal experiments, using finer gradations of robot appearances and behavior are required in order to give more data sample points and to provide more extensive evidence, which can then be used to refine the parameters which govern human perception of robot appearance and behavior and how these can be applied to developing principles for robot aesthetics. The reasons for participants' preferences were only touched upon in this study and it is seen as desirable that future work should explore participants' reasons for their preferences and responses in more detail. A longitudinal study with repeated exposure to robots with different appearances was carried out in 2006 in the Robot House and results are currently being analyzed, preliminary results can be found in Koay et al. (2007) and Syrdal et al.(2007).

We hope that the methods used here and the results gained yield useful insights into how to calibrate robot appearance and behavior so that owners and users of domestic or companion robots in future will be less disaffected due to design feature limitations which do not live up to their initial expectations.

\section{ACKNOWLEDGMENTS}

The work described in this paper was conducted within the EU Integrated Project COGNIRON ("The Cognitive Robot Companion") and was funded by the European Commission Division FP6-IST Future and Emerging Technologies under Contract FP6-002020.

Thanks go to Steve Ho for starring in the videos used in the trial. Thanks also go to all our colleagues at the University of Hertfordshire who helped administer the trials, but in particular to Sarah Woods for her help.

\section{REFERENCES}

Albright, L., Kenny, D. A., and Malloy, T. E. 1988. Consensus in personality judgments at zero acquaintance. Journal of Personality and Social Psychology, 55(3), 387-395.

Asch, S. E. ((1946). Forming impressions of personality. Journal of Abnormal and Social Psychology, 41, 258-290.

Asquith, P. J. 1997. Why anthropomorphism is not metaphor: Crossing concepts and cultures in animal behavior studies. In R. W. Mitchell, N. S. Thompson and H. L. Miles (Eds.), Anthropomorphism, anecdotes and animals. Albany, New York: State University of New York Press, pp. $22-34$.

Ball, G., \& Breese, J. (2000). Emotion and personality in a conversational agent. In J. Cassell, J. Sullivan, S. Prevost \& E. Churchill (Eds.), Embodied conversational agents. Cambridge, MA: MIT Press.

Bartneck C., Nomura T., Kanda T., Suzuki T. and Kennsuke K. 2005. A cross-cultural study on attitudes towards robots. Proceedings of the HCI International 2005, Las Vegas.

Bethal C. L., Murphy R. R.. 2006. Affective Expression in Appearance-Constrained Robots. In Proceedings of ACM SIGCHI/SIGART 2nd Conference on Human Robot Interaction (HRI '06). Salt Lake City, Utah, US, pp. 327-328.

Bickmore, T. W.; \& Cassell, J. (2005). Social Dialogue with Embodied Conversational Agents. In J. v. Kuppevelt, L. Dybkjaer \& N. Bernsen (Eds.), Natural, Intelligent and Effective Interaction with Multimodal Dialogue Systems (pp. 23-54). New York: Kluwer Academic. 
Blow M., Dautenhahn K., Appleby A., Nehaniv C. L., and Lee D. 2006. The Art of Designing Robot Faces - Dimensions for Human-Robot Interaction. In Proceedings of HRI '06, Salt Lake City, Utah, USA, pp. 321-332.

te Boekhorst R., Walters M. L., Koay K. L., Dautenhahn K., Nehaniv C. L.. 2005. A Study of a Single Robot Interacting with Groups of Children in a Rotation Game Scenario. . In Proceedings of IEEE CIRA 2005. ESpoo, Finland, pp. 35-40.

Borkenau, P., and Liebler, A. 1992. Trait inferences: Sources of validity at zero acquaintance. Journal-of-Personality-and-Social-Psychology, 62(4), 645657 .

Breazeal C. L.. 2002. Designing Sociable Robots. Cambridge, MA: MIT Press.

Brenton H., Gillies M., Ballin D., Chattin D.. 2005. The Uncanny Valley: Does it exist?. In Proceedings of Conference of Human Computer Interaction, Workshop on Human animated Character Interaction. . Napier University, Edinburgh.

Bruce A., Nourbakhsh I., Simmons R.. 2002. The Role of Expresssiveness and Attention in Human-Robot Interaction. In Proceedings of IEEE International Conference on Robotics and Automation (ICRA 2002). Washington DC, USA.

Burgoon J. K., \& Jones S. B. 1976. Toward a Theory of Personal Space Expectations and Their Violations. Human Communication Research 2(2), p. 131146

Dautenhahn K.. 2002. Design spaces and niche spaces of believable social robots. In Proceedings of the 11th Annual International Workshop on Robot and Human Interactive Communication (RO-MAN 02). Berlin, Germany, pp. 192-197.

Dautenhahn K. Robots We Like to Live With? - A Developmental Perspective on a Personalized, Life-Long Robot Companion. Proceedings of the 13th IEEE International Workshop on Robot and Human Interactive Communication (RO-MAN 2004), pp. 17-22., 2004.

Dautenhahn K., Woods S. N., Kaouri C., Walters M. L., Koay K. L., Werry I.. 2005. What is a Robot companion - Friend, Assistant or Butler? In Proceedings of IEEE RSJ International Conference on Intelligent Robot Systems (IROS'05). Edmonton, Canada, pp. $1488-1493$.

Dautenhahn K., Walters M. L., Woods S. N., Koay K. L., Nehaniv C. L., Sisbot E. A., Alami R., Simeon T.. 2006. How may I serve you? A robot companion approaching a seated person in a helping context. In Proceedings of ACM SIGCHI/SIGART 2nd Conference on Human Robot Interaction (HRI '06). Salt Lake City, Utah, USA, pp. 172-179.

Davis, H. 1997. Animal cognition versus animal thinking: The anthropomorphic. In R. W. Mitchell, N. S. Thompson and H. L. Miles (Eds.), Anthropomorphism, anecdotes, and animals. Albany, State University of New York Press.

Deaux, K., Dane, F. C., and Wrightsman, L. S. 1993. Social psychology in the '90s (6th ed.). Pacific Grove, CA: Brooks/Cole.

DiSalvo C. and F. Gemperle. 2003. From seduction to fulfillment: the use of anthropomorphic form in design," Proceedings of the 2003 international conference on Designing pleasurable products and interfaces, June 23-26, 2003, Pittsburgh, PA, USA, 2003.

DiSalvo C., Gemperle F., Forlizzi J., and Kiesler S. 2002. All robots are not created equal: the design and perception of humanoid robot heads. Proceedings of the conference on Designing Interactive Systems: Processes, Practices, Methods, and Techniques, June 25-28, 2002, London, England.

Dryer D. C. 1999. Getting Personal with Computers: How to Design Personalities for Agents. Applied Artificial Intelligence 13:3, pp. 273 - 295.

Duffy B. R. 2003. Anthropomorphism and The Social Robot. Robotics and Autonomous Systems 42, pp.177-190.

Ferber D. 2003. The Man Who Mistook His Girlfriend For A Robot. Popular Science September 2003.

Fong T., Nourbakhsh I., Dautenhahn K. 2003. A Survey of Socially Interactive Robots. Robotics and Autonomous Systems 42(4-3), p. 143-166.

Friedman B., Khan P. H., Hagman J.. 2003. Hardware Companions? - What Online AIBO Discussion Forums Reveal about the Human-Robotic Relationship. In Proceedings of the CHI 2003 Conference on Human Factors in Computing Systems. Ft Lauderdale, Florida, USA, pp. $273-279$.

Gill, A. J., Oberlander, J., and Austin, E. (2006). Rating e-mail personality at zero acquaintance. Personality-and-Individual-Differences, $40(3), 497-507$.

Gillespie D. L., Leffler A. 1983. Theories of Nonverbal Behavior: A Critical Review of Proxemics Research. Sociological Theory 1, pp. $120-154$.

Gockley R. and Mataric' M.. 2006. Encouraging Physical Therapy Compliance with a HandsOff Mobile Robot. In Proceedings of ACM SIGCHI/SIGART 2nd Conference on Human Robot Interaction (HRI '06). Salt Lake City, USA, pp. 150-155.

Goetz J. and Kiesler S., Powers. A.. 2003. Matching Robot Appearance and Behavior to Tasks to Improve Human-Robot Cooperation. In Proceedings of the 12th IEEE International Workshop on Robot and Human Interactive Communication. Berkeley, CA, USA, pp. 55 - 60.

Goldberg, L. R. (1999). A broad-bandwidth, public domain, personality inventory measuring the lower-level facets of several five-factor models. Personality Psychology in Europe, 7, 7-28.

Gong, L., Nass, C. (2007). When a Talking-Face Computer Agent is Half-Human and Half-Humanoid: Human Identity and Consistency Preference. Journal of Human Communication Research. 33(2), 163-193.

Hall E. T.. 1966. The Hidden Dimension. New York: Doubleday.

Hall E. T. 1968. Proxemics.. Current Anthropology 9(2-3), pp. 83-108. 
Hanson D.. 2006. Exploring the Aesthetic Range for Humanoid Robots. In Proceedings of Cognitive Science (CogSci 2006) Workshop on Android Science. Vancouver, BC, Canada, .

Hanson D., Olney A., Pereira I. A. and Zielke M.. 2005. Upending the uncanny valley. In Proceedings of the American Association for Artificial Intelligence (AAII) Conference. Pittsburgh, PA, USA.

Hinds P. J., Roberts T. L. andJones H. 2004. Whose Job Is It Anyway? A Study of Human-Robot Interaction in a Collaborative Task. Human Computer Interaction 19, pp. 151-181.

Ishiguro H. 2007. Scientific Issues Concerning Androids. The International Journal of Robotics Research 26, pp. 101-117.

Kanda T., Hirano T. and Eaton D. 2004. Interactive Robots as Social Partners and Peer Tutors for Children: A Field Trial. Human-Computer Interaction 19, pp. 61-84.

Kanda T., Kamasima M., Imai M., Ono T., Sakamoto D., Ishiguro H., Anzai Y. 2007. A Humanoid Robot that Pretends to Listen to Route Guidance from a Human. Autonomous Robots 22(1), .

Khan Z. 1998. Attitudes Towards Intelligent Service Robots. Technical report, IPLab, NADA, Royal Institute of Technology. TRITA-NA-E98421 IPLab-154.

Kiesler S. and Goetz J. 2000. Machine Trait Scales for Evaluating Mechanistic Mental Models of Robots and Computer-Based Machines, White paper, www.peopleandrobots.org/hri/images/Machine scales.pdf. Site last accessed January 2007

Koay, K. L.;Syrdal, D. S.;Walters, M. L.; \& Dautenhahn, K. In press. Living with Robots: Investigating the Habituation Effect in Participants' Preferences during a Longitudinal Human-Robot Interaction Study. IEEE International Symposium on Robot and Human Interactive Communication(Ro-man), Jeju Island, Korea.

Lambert D.. 2004. Body Language. New York: HarperCollins.

Lee S., Kiesler S.. 2005. Human Mental Models of Humanoid Robots. In Proceeedings of the 2005 International Conference on Robotics and Automation (ICRA 05). Barcelona, Spain,pp. 2767- 2772.

Li S., Wrede B., Sagerer. G.. 2006. A dialog System for Comparitive User Studies on Robot Verbal Behavior. In Proceedings of The 15th IEEE International Symposium on Robot and Human Interactive Communication (RO-MAN06), pp. 129-134.

Luczak, H., Roetting, M., and Schmidt, L. (2003). Let's talk: Anthropomorphization as means to cope with stress of interacting with technical devices. Ergonomics, 46(13/14), 1361-1374.

MacDorman K. F.. 2005. Androids as an experimental apparatus: Why is there an uncanny valley and can we exploit it?. In Proceedings Of the CogSci 2005 Workshop: Toward Social Mechanisms of Android Science. Stresa, Italy, pp. 106-118.

MacDorman, K., Ishiguro, H. (2006). The Uncanny Advantage of Using Androids in Cognitive and Social Science Research, Interaction Studies, 7(3), 297-337.

Matthews, G., Deary, I. J., and Whiteman, M. C. (2003). Personality traits. Cambridge, UK: Cambridge University Press.

Minato T., Shimada M., Ishiguro H., Itakura S.. 2004. Development of an Android Robot for Studying Human-Robot Interaction, Innovations in Applied Artificial Intelligence. In Proceedings of the 17th International Conference on Industrial and Engineering Applications of Artificial Intelligence and Expert Systems (IEA/AIE 04), pp. 424-434.

Minato T., MacDorman K. F., Shimada M., Itakura S., Lee K., Ishiguro H.. 2004. Evaluating Humanlikeness by Comparing Responses Elicited by an Android and a Person. In Proceedings of the Second International Workshop on Man-Machine Symbiotic Systems. Kyoto, Japan., pp. 373-383.

Mori M. 1970. Bukimi no tani (The uncanny valley). Energy 7(4), pp. 33-35.

Nass, C.;Moon, Y.;Fogg, B. J.; \& Reeves, B. 1995. Can computer personalities be human personalities? International-Journal-of-Human-Computer-Studies 43(2): 223-239.

Nass, C. I.;Lombard, M.;Henriksen, L.; \& Steuer, J. 1995. Anthropocentrism and computers. Behaviour-and-Information-Technology 14(4): $229-238$.

Norman D.. 2001. How might humans interact with robots. In Proceedings of the DARPA/NSF Workshop on Human-Robot Interaction. San Luis Obispo, CA.

Nomura T. and Kanda T. 2003. On proposing the concept of robot anxiety and considering measurement of it. Proceedings, 12th IEEE International Workshop on Robot and Human Interactive Communication(RO-MAN 2003), pp. 373-378.

Nomura T., T. Kanda, and Y. Suzuki. 2006.Experimental Investigation into Influence of Negative Attitudes toward Robots on Human-Robot Interaction. In $\mathrm{AI}$ and Society, 20.

Reeves B., Nass C.. 1996. The Media Equation: How People Treat Computers, Television, and New Media Like Real People and Places. Cambridge University Press. 
Robins B., Dautenhahn K., te Boerkhorst R., and Billard A., 2004. Robots as assistive technology - does appearance matter? In Proceedings of 13th IEEE International Workshop On Robot And Human Interactive Communication (RO-MAN 2004), pp. $277-282$.

Scopelliti M., Giuliani M. V., D'Amico A. M., Fornara F.. 2004. If I had a robot at home.... Peoples' representation of domestic robot. In Designing a more inclusive world. S. Keates, J. Clarkson, P. Langdon, P. Robinson (Ed.). pp. 257-266.

Severinson-Eklundh K., Green A., Huutenrauch H. 2003. Social and /colaborative Aspects of Interaction with a Service Robot. Robotics and Autonomous /systems 42, pp. 223-234.

Stratton L. O., Tekippe D. J., Flick G. L. 1973. Personal Space and Self Concept. Sociometry 36, pp. 424-429.

Syrdal D. S., Dautenhahn K., Woods S. N., Walters M. L., Koay K. L.. 2006. Doing the Right Thing Wrong' - Personality and Tolerance to Uncomfortable Robot Approaches. . In Proceedings of The 15th IEEE International Symposium on Robot and Human Interactive Communication (RO-MAN06). University of Hertfordshire, UK.

Syrdal, D. S.;Dautenhahn, K.;Woods, S.;Walters, M.; \& Koay, K. L. 2007. Looking Good? Appearance Preferences and Robot Personality Inferences at Zero Acquaintance. Multidisciplinary Collaboration for Socially Assistive Robotics: Papers from the AAAI Spring Symposium -Technical Report SS-0707: 86-92.

Syrdal, D. S.;Koay, K.-L.;Walters, M. L.; \& Dautenhahn, K. 2007. A personalised robot companion? The role of individual differences on spatial preferences in HRI scenarios. IEEE International Symposium on Robot and Human Interactive Communication(Ro-man), Jeju Island, Korea.

Walters M. L., Woods S. N., Koay K. L., Dautenhahn K. 2005. Practical and Methodological Challenges in Designing and Conducting Human-Robot Interaction Studies. In Proceedings of the AISB'05 Symposium on Robot Companions Hard Problems and Open Challenges in Human-Robot Interaction. UK, pp. 110-119.

Walters M. L., Dautenhahn K., Koay K. L., Kaouri C., te Boekhorst R., Nehaniv C. L., Werry I., Lee D.. 2005. Close encounters: Spatial distances between people and a robot of mechanistic appearance. In Proceedings of IEEE-RAS International Conference on Humanoid Robots (Humanoids2005). Tsukuba, Japan, pp. 450-455.

Walters M. L., Dautenhahn K., te Boekhorst R., Koay K. L., Kaouri C., Woods S. N., Nehaniv C. L., Lee D., Werry I.. 2005. The Influence of subjects'Personality Traits on Personal Spatial Zones in a Human-Robot Interaction Experiment. In Proc. IEEE Ro-man 2005, 14th IEEE International Workshop on Robot and Human Interactive Communication (RoMan05). Nashville, USA, pp. 347-352.

Walters M. L., Dautenhahn K., te Boekhorst R., Koay K. L.. 2007. Exploring the Design Space of Robot Appearance and Behaviour in an AttentionSeeking 'Living Room' Scenario for a Robot Companion. In Proceedings of IEEE-Artificial Life (Alife 07). Honolulu, Hawaii, USA, pp. $341-347$.

Walters M. L., Koay K. L., Dautenhahn K., te Boekhorst R., Syrdal D. S.. 2008. Human Approach Distances to a Mechanical-Looking Robot with Different Robot Voice Styles. In Submitted for publication. .

Woods S. N., Dautenhahn K., Schulz J.. 2004. The Design Space of Robots: Investigating Children's Views. In Proceedings of 13th IEEE International Workshop on Robot and Human Interactive Communication (RO-MAN 04). Kurashiki, Okayama Japan, pp. 47-52.

Woods S. N., Walters M. L., Koay K. L., Dautenhahn K.. 2006. Comparing Human Robot Interaction Scenarios Using Live and Video Based Methods: Towards a Novel Methodological Approach. In Proceedings of The 9th International Workshop on Advanced Motion Control (AMC'06). Istanbul, Turkey, pp. $750-755$.

Woods S. N., Walters M. L., K.oay K. L., Dautenhahn K.. 2006. Methodological Issues in HRI: A Comparison of Live and Video-Based Methods in Robot to Human Approach Direction Trials. In Proceedings of The 15th IEEE International Symposium on Robot and Human Interactive Communication (ROMAN06). Hertfordshire, UK, pp. 51-58.

Woods S. N., Dautenhahn K., Kaouri C., te Boekhorst R., Koay K. L., Walters M. L. 2007. Are Robots Like People? - Relationships between Participant and Robot Personality Traits in Human-Robot Interaction. Interaction Studies 8(3), pp. 281-305.

Yan, C., Peng, W., Lee, K. M., \& Jin, S. (2004). Can robots have personality?An empirical study of personality manifestation, social responses, and social presence in human-robot interaction. Proceedings of the 54th Annual Conference of the International Communication Association.

Zebrowitz L. A., Hall J. A., Murphy N. A., Rhodes G. 2004. Looking Smart and Looking Good: Facial Cues to Intelligence and Their Origins. Personality and Social Psychology Bulletin 28 , pp. 238-249. 


\section{FOOTNOTES}

M. L. Walters, D. S. Syrdal, K. Dautenhahn, R. te Boekhorst, K. L. Koay. All authors affiliated to University of Adaptive Systems Research Group, School of Computer Science, University of Hertfordshire, College Lane, Hatfield, Hertfordshire, AL10 9AB, UK.

iiThe HRI study which provides the basis of the research presented in this article has been presented in preliminary form in two conference papers, Walters et al. (2007d) and Syrdal et al. (2007a). 